\title{
COMBINATORIAL ASPECTS OF CONTINUED FRACTIONS
}

\author{
P. FLAJOLET \\ IRIA, 78150 Rocquencourt, France
}

Received 23 March 1979

Revised 11 February 1980

\begin{abstract}
We show that the universal continued fraction of the Stieltjes-Jacobi type is equivalent to the characteristic series of labelled paths in the plane. The equivalence holds in the set of series in non-commutative indeterminates. Using it, we derive direct combinatorial proofs of continued fraction expansions for series involving known combinatorial quantities: the Catalan numbers, the Bell and Stirling numbers, the tangent and secant numbers, the Euler and Eulerian numbers... . We also show combinatorial interpretations for the coefficients of the elliptic functions, the coefficients of inverses of the Tchebycheff, Charlier, Hermite, Laguerre and Meixner polynomials. Other applications include cycles of binomial coefficients and inversion formulae. Most of the proofs follow from direct geometrical correspondences between objects.
\end{abstract}

\section{Introduction}

In this paper we present a geometrical interpretation of continued fractions together with some of its enumerative consequences. The basis is the equivalence between the characteristic series of positive labelled paths in the plane and the universal continued fraction of the Jacobi type. The equivalence can be asserted in the strong form of an equality in the set of formal series in non-commutative variables. Using this framework leads to a direct "non computational" proof.

Section 1 contains the proof of this equivalence (Theorem 1) together with a combinatorial interpretation of the Stieltjes matrix and the Rogers polynomials. It extends some previous results of Touchard [27] and independent works of Jackson [14], Read [32] and the author [9].

Section 2 is devoted to direct derivations of continued fraction expansions for generating series of known combinatorial quantities. Indeed the expansions of series relative to many classical combinatorial quantities have integer coefficients obeying simple laws whose origin can be combinatorially accounted for. In the case of path enumerations, these expansions follow as direct consequences of our basic theorem. In other contexts, the proof is achieved by utilizing Theorem 1 in conjunction with what we name systems of path diagrammes. Path diagrammes are related to the weighted ballot sequences of Rosen [24]; they have been used systematically by Françon and Viennot $[12,13]$ to enumerate various classes of permutations.

We show here that one system of path diagrammes bijectively corresponds to set partitions. From this, a set of continued fraction expansions (Theorem 2) is 
derived for series involving the Bell numbers, the Stirling numbers of the second kind, the odd factorial numbers and other related quantities. A second set (Theorem 3) is obtained by exploiting the Françon-Viennot's correspondence and some of its variants $[12,13]$ between another system of path diagrammes and permutations. Using it, we derive continued fraction expansions for series involving the factorial numbers, the Euler numbers, the Eulerian numbers, the Stirling numbers of the first kind and other quantities; extensions include the generalized Eulerian and Euler numbers of order $k$. Conversely, Theorem 1 makes it possible to interpret combinatorially those Jacobi type continued fractions which have integer coefficients. As an application we show that the coefficients of the elliptic functions $\mathrm{cn}, d n$ count alternating permutations partitioned according to the number of minima of even value (Theorem 4). Finally we show as a continuation of remarks of Section 1, how to derive generating series for Carlitz's cycles of binomial coefficients [2].

Section 3 is devoted to the enumerative properties of convergents of continued fractions. The denominator polynomials appear in a number of enumerating series for paths and diagrammes. Their classical orthogonality relations underlie inversion formulae which have the following interpretation: the matrix formed with the coefficients of the denominator polynomials is the inverse of the Stieltjes matrix. Considering in particular the convergents of those continued fractions introduced in Section 2 for enumerative purposes leads to fractions involving the classical Hermite, Charlier, Laguerre and Meixner polynomials for which combinatorial interpretations are given (Theorem 5). In particular, we show that the Taylor coefficients of inverses of these polynomials enumerate various classes of permutations.

It should be pointed out that all the continued fraction expansions we derive here obtain as a direct consequence of geometrical correspondences without any computation over generating functions. Such an attitude towards enumeration problems originates in the works of Foata and Schützenberger; quoting from [11]: "Plus important nous semble la démonstration du fait que toutes les identités classiques... sont seulement la traduction de propriétés très simples des morphismes d'ensembles totalement ordonnés". Strikingly enough, as we see here, almost all the classical expansions having integer coefficients receive simple combinatorial interpretations.

\section{Labelled paths and continued fractions}

\subsection{The basic equivalence}

In this section, we prove the basic equivalence theorem relating the characteristic series of certain labelled paths in the plane to the universal Stieltjes-Jacobi continued fractions.

Paths we wish to consider here are positive paths in the $x-y$ plane, which 
consist of only three types of steps: rises, levels and falls. More precisely we start with three step vectors $a=(1,1), b=(1,-1), c=(1,0)$ called respectively rise vector, fall vector and level vector; to each word $u=u_{1} u_{2} \cdots u_{n}$ on the alphabet $\{a, b, c\}$ is associated a sequence of points $M_{0} M_{1} \cdots M_{n}$ where $M_{0}=0=(0,0)$ and for each $j$ s.t. $1 \leqslant j \leqslant n, O M_{j}=O M_{j-1}+u_{j}$; in other words for $M_{j}=\left(x_{j}, y_{j}\right)$ and $u_{j}=\left(s_{j}, t_{j}\right)$ :

(i) $\left(x_{0}, y_{0}\right)=(0,0)$,

(ii) $\left(x_{j}, y_{j}\right)=\left(x_{j-1}, y_{j-1}\right)+\left(s_{j}, t_{j}\right)$ for $1 \leqslant j \leqslant n$.

The number $n$ is the length of $u$; for each $j$, the number $y_{j}$ is the height of point $M_{j}$; finally the height of the sequence $u$ (or equivalently of $M_{0} M_{1} \cdots M_{n}$ ) is defined as $\max _{0 \leqslant j \leqslant n}\left\{y_{j}\right\}$ and is denoted by $h(u)$ or $h\left(M_{0} M_{1} \cdots M_{n}\right)$.

We wish to restrict attention to certain sequences called positive paths or simply paths: these are sequences such that all the points in the associated sequence have a non-negative $y$-coordinate. We let $\mathscr{P}^{+}$denote the set of positive paths

$$
\begin{gathered}
\mathscr{P}^{+}=\left\{\left.u_{1} u_{2} \cdots u_{n} \in\{a, b, c\}^{*}|j: 1 \leqslant j \leqslant n,| u_{1} u_{2} \cdots u_{j}\right|_{a} \geqslant\left|u_{1} u_{2} \cdots u_{j}\right|_{b}\right. \\
\text { and } \left.\left|u_{1} u_{2} \cdots u_{n}\right|_{a}=\left|u_{1} u_{2} \cdots u_{n}\right|_{b}\right\}
\end{gathered}
$$

where $|x|_{c}$ denotes the number of occurrences of $c$ in $x$.

It proves convenient for later applications to consider the empty word as a positive path of height and length equal to zero.

We now define labelled paths in which each step is indexed with the height of the point from which it starts: if $u=u_{1} \cdots u_{n}$ is a positive path, and if $M_{0} M_{1} \cdots M_{n}$ with $M_{j}=\left(x_{j}, y_{j}\right)$ is the associated sequence of points, the labelling of $u, \lambda(u)$ is defined as a word over the infinite alphabet $X=$ $\left\{a_{0}, a_{1}, a_{2}, \ldots\right\} \cup\left\{b_{1}, b_{2}, \ldots\right\} \cup\left\{c_{0}, c_{1}, c_{2}, \ldots\right\}$ by: $\lambda(u)=v_{1} v_{2} \cdots v_{n}$ where for $1 \leqslant$ $j \leqslant n: v_{j} \in X$, and

(i) if $u_{j}=a$, then $v_{j}=a_{y_{j-1}}$,

(ii) if $u_{j}=b$, then $v_{j}=b_{y_{j-1}}$,

(iii) if $u_{j}=c$, then $v_{j}=c_{y_{j-1}-1}$.

We let $\mathscr{P}=\lambda\left(\mathscr{P}^{+}\right)$denote the set of labelled paths. The labelling operation and the geometrical representation of paths are exemplified in Fig. 1.

We now need a few concepts from the theory of series in non-commutative variables, whose introduction in the context of enumerative problems is due to

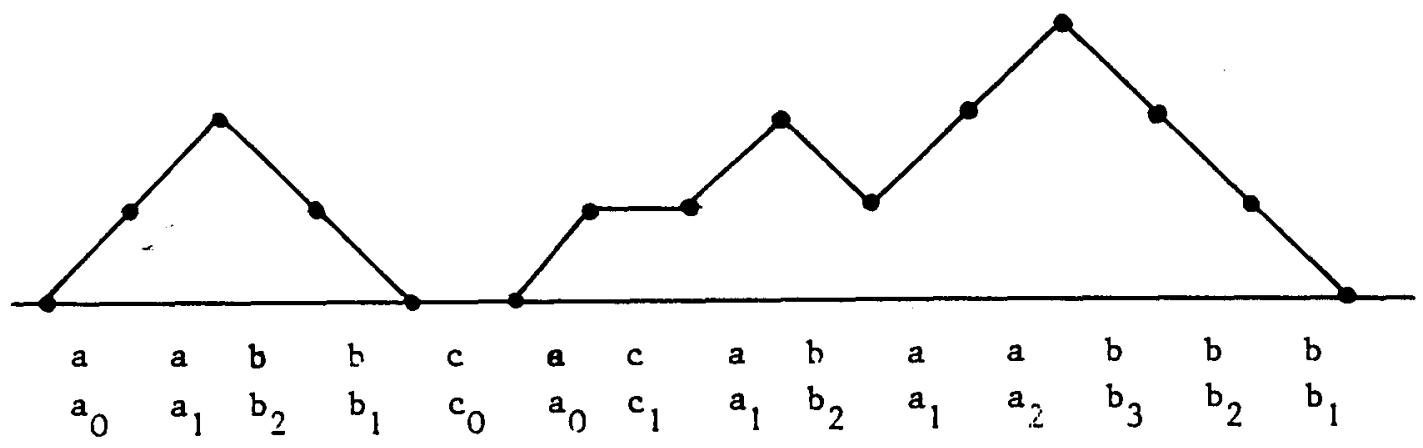

Fig. 1 
Schützenberger [25] (see also Raney [20] for applications to Lagrange's inversion formula, or Cori [6] in connection with planar graph enumerations).

We consider the monoid algebra ${ }^{1} C\langle\langle X\rangle$ of formal series on the set of non-commutative variables (alphabet) $X$ with coefficients in the field of complex numbers. An element of $C\langle\langle X\rangle$ can be written as

$$
s=\sum_{u \in X^{*}} s_{u} \cdot u
$$

Sums and Cauchy products are defined in the usual way:

$$
\begin{aligned}
s+t & =\sum_{u \supset X^{*}}\left(s_{u}+t_{u}\right) \cdot u \\
s \cdot t & =\sum_{u \in X^{*}}\left(\sum_{v w=u} s_{v} t_{w}\right) \cdot u .
\end{aligned}
$$

The valuation of a series is defined by

$$
\operatorname{val}(s)=\min \left\{|u| ; s_{u} \neq 0\right\}
$$

with $|u|$ denoting the length of $u$; conventionally val $(0)=+\infty$. Convergence in $C\left\langle\langle X\rangle\right.$ is introduced as follows: a sequence $\left\{s_{n}\right\}_{n \geqslant 0}$ where each $s_{n}$ is in $C\langle\langle X\rangle$, has limit $s$ iff

$$
\lim _{n \rightarrow \infty} \operatorname{val}\left(s-s_{n}\right)=+\infty
$$

In other words, the sequence $\left\{s_{n}\right\}$ has limit $s$ iff the coefficients of the $s_{n}$ progressively stabilize starting with terms of lower order. This induces a notion of summability for infinite sequences.

Multiplicative inverses exist for series having a constant term different from zero; this is in particular the case for series of the form $(1-u)$ with $\operatorname{val}(u)>0$, for which we have

$$
(1-u)^{-1}=\sum_{k \geqslant 0} u^{k}
$$

The element $(1-u)^{-1}$ is known as the quasi-inverse of $u$.

Finally for every set of words $S \subset X^{*}$ we define the characteristic series of $S$, which we denote char $(S)$, by

$$
\operatorname{char}(S)=\sum_{u \in S} u
$$

For $E, F$ subsets of $X^{*}$, let $E+F$ be an alternative notation for $E \cup F$. Let $E \cdot F$ be the extension to sets of the catenation operation on words and let $E^{*}=$ $\varepsilon+E+E \cdot E+E \cdot E \cdot E+\cdots$ with $\varepsilon$ the empty word. We shall make use of the following classical lemma.

${ }^{1}$ For extensive definitions, we refer the reader to standard treatises on the subject for instance [8]. 
Lemma 1. Let $E, F$ be subsets of $X^{*}$. Then

(i) $\operatorname{char}(E+F)=\operatorname{char}(E)+\operatorname{char}(F)$ provided $E \cap F=\emptyset$,

(ii) $\operatorname{char}(E \cdot F)=\operatorname{char}(E) \cdot \operatorname{char}(F)$ provided $E \cdot F$ has the unique factorization property, i.e. $\forall u, u^{\prime} \in E \forall v, v^{\prime} \in F u v=u^{\prime} v^{\prime}$ implies $u=u^{\prime}$ and $v=v^{\prime}$;

(iii) $\operatorname{char}\left(E^{*}\right)=(1-\operatorname{char}(E))^{-1}$ provided the following two conditions hold:

$E^{j} \cap E^{k}=\emptyset$ for all $j, k$ with $j \neq k$,

each $E^{k}$ has the unique factorization property.

Lemma 1 thus makes it possible to translate operations on sets of words into corresponding operations on series provided certain non ambiguity conditions are satisfied. We can now state

Theorem 1. Let $C^{[h]}, h \geqslant 0$, be the formal power series:

$$
\begin{aligned}
& C^{[h]}=\frac{1}{1-c_{0}-\frac{a_{0} \mid b_{1}}{1-c_{1}-\frac{a_{1} \mid b_{2}}{\cdots}}} \\
& 1-c_{h-1}-\frac{a_{h-1} \mid b_{h}}{1-c_{h}}
\end{aligned}
$$

where $(u \mid v) / w$ denotes $u w^{-1} v$. Then

(i) the sequence $\left\{C^{[h]}\right\}_{h \geqslant 0}$ converges, its limit defining the infinite continued fraction:

$$
\lim _{h \rightarrow \infty} C^{[h]}=\frac{1}{1-c_{0}-\frac{a_{0} \mid b_{1}}{1-c_{1}-\frac{a_{1} \mid b_{2}}{1-c_{2}-\frac{a_{2} \mid b_{3}}{\cdots}}}}
$$

(ii) the characteristic series of labelled paths is equal to this infinite continued fraction:

$$
\operatorname{char}(\mathscr{P})=\frac{1}{1-c_{0}-\frac{a_{0} \mid b_{1}}{1-c_{1}-\frac{a_{1}}{1-c_{2}-\frac{a_{2} \mid b_{3}}{\ldots}}}}
$$

Proof. For each $h \geqslant 0$, we define the set $X^{[h]}=\left\{c_{0}, c_{1}, \ldots, c_{h}\right\} \cup$ $\left\{a_{0}, a_{1}, \ldots, a_{h-1}\right\} \cup\left\{b_{1}, b_{2}, \ldots, b_{h}\right\}$. The set $\mathscr{P}^{[h]}=\mathscr{P} \cap\left(X^{[h]}\right)^{*}$ is also the set of all labelled paths with height $\leqslant h$. We first show that

$$
\operatorname{char}\left(\mathscr{P}^{[h]}\right)=C^{[h]} \text {. }
$$

For each $h$, the set $\mathscr{P}^{[h]}$ is a regular or rational set [8]. Indeed we have

$$
\begin{aligned}
& \mathscr{P}^{[0]}=\left(c_{0}\right)^{*}, \\
& \mathscr{P}^{[1]}=\left(c_{0}+a_{0} c_{1}{ }^{*} b_{1}\right)^{*}, \\
& \mathscr{P}^{[2]}=\left(c_{0}+a_{0}\left(c_{1}+a_{1} c_{2}{ }^{*} b_{2}\right)^{*} b_{1}\right)^{*},
\end{aligned}
$$


and by induction we see that for each $h \geqslant 0$ :

$$
\mathscr{P}^{[h+1]}=\mathscr{P}^{[h]} \circ \sigma^{[h]} \text {. }
$$

where $\sigma_{n}$ is the substitution: $c_{h} \rightarrow\left(c_{h}+a_{h} c_{h+1}^{*} b_{h+1}\right)$. We thus have for each $h \geqslant 0$ the following description of $\mathscr{P}^{[h]}$ :

$$
\mathscr{P}^{[h]}=\left(c_{0}+a_{0}\left(c_{1}+a_{1}\left(c_{2}+\cdots\left(c_{h-1}+a_{h-1} c_{h}^{*} b_{h}\right)^{*} \cdots b_{2}\right)^{*} b_{1}\right)^{*} .\right.
$$

The series $C^{[h]}$ is obtained from $\mathscr{P}^{[h]}$ by replacing each of the set-theoretic operations,$+ \cdot$, star operation by the corresponding series operations:,$+ \cdot$, quasi-inverse. The equality $C^{[h]}=\operatorname{char}\left(\mathscr{P}^{[h]}\right.$ follows from the observation that all operations in the above expression for $\mathscr{P}^{[h]}$ are unambiguous; this fact is itself readily proved by introducing the sets:

$$
\begin{aligned}
& \mathscr{P}^{[h, h]}=c_{h}^{*}, \\
& \mathscr{P}^{[h, h-1]}=\left(c_{h-1}+a_{h-1} c_{h}^{*} b_{h}\right)^{*} \cdots,
\end{aligned}
$$

and by checking that each of the $\mathscr{P}^{[h, h-k]}$ has the unique factorization property.

To complete the proof of the theorem-part (ii)—, we notice the following chain of inclusions:

$$
\mathscr{P}^{[0]} \subset \mathscr{P}^{[1]} \subset \mathscr{P}^{[2]} \cdots \subset \mathscr{P},
$$

together with the property of the $C^{[h]}=\operatorname{char} \mathscr{P}^{[h]}$ :

$$
\operatorname{val}\left(C^{[h]}-C^{[h-1]}\right)=2 h,
$$

which simply expresses the fact that a path of height $h$ must have length $\geqslant 2 h$ (the shortest path of height $h$ is $\left.a_{0} a_{1} \cdots a_{h-1} b_{h} \cdot b_{2} b_{1}\right)$. We thus have

$$
\lim _{h \rightarrow \infty} C^{[h]}=\operatorname{char}(\mathscr{P}),
$$

the convergence being monotonic.

\subsection{Continued fractions and power series: the Rogers polynomials}

We are here considering connections between power series and continued fractions. After Theorem 1, the series $\operatorname{char}(\mathscr{P})$ thus appears as the noncommutative analogue of the Jacobi type continued fraction ( $J$-Fraction) which is usually taken under the form $[19,30]$ :

$$
\frac{1}{1-c_{0} z-\frac{b_{1} z^{2}}{1-c_{1} z-\frac{b_{2} z^{2}}{\cdots}}}
$$

However here for convenience we define it to be

$$
J(X, z)=\frac{1}{1-c_{0} z-\frac{a_{0} b_{1} z^{2}}{1-c_{1} z-\frac{a_{1} b_{2} z^{2}}{\cdots}},}
$$


also denoted $J(z)$. Similarly, the Stieltjes type continued fraction appears when we set formally the $c_{j}$ 's to 0 . If we let $X^{\prime}=\left\{a_{0}, a_{1}, \ldots, b_{1}, b_{2}, \ldots\right\}$, we define it as

$$
S\left(X^{\prime}, z\right)=\frac{1}{1-\frac{a_{0} b_{1} z^{2}}{1-\frac{a_{1} b_{2} z^{2}}{1-\frac{a_{2} b_{3} z^{2}}{\cdots}}}} .
$$

Each of these fractions has a power series expansion in $z$ :

$$
J(X, z)=\sum_{n \geqslant 0} R_{n} z^{n} \text { and } S\left(X^{\prime}, z\right)=\sum_{n \geqslant 0} R_{n}^{\prime} z^{n} .
$$

These expansions define quantities $\left\{R_{n}\right\}_{n \geqslant 0}$ and $\left\{R_{n}^{\prime}\right\}_{n \geqslant 0}$ that are polynomials in $X$ and $X^{\prime}$ respectively; we name the $R_{n}$ Jacobi-Rogers polynomials, and the $R_{n}^{\prime}$ Stieltjes-Rogers polynomials. These polynomials have been first considered by Rogers [23]. A simple computation shows:

$$
\left\{\begin{array}{l}
R_{0}=1 ; R_{1}=c_{0} ; R_{2}=c_{0}^{2}+a_{0} b_{1} ; R_{3}=c_{0}^{3}+c_{0} a_{0} b_{1}+a_{0} c_{1} b_{1}+a_{0} b_{1} c_{0} \\
R_{0}^{\prime}=1 ; R_{1}^{\prime}=0 ; R_{2}^{\prime}=a_{0} b_{1} ; R_{3}^{\prime}=0 ; R_{4}^{\prime}=a_{0} a_{1} b_{2} b_{1}+a_{0} b_{1} a_{0} b_{1} ; \ldots
\end{array}\right.
$$

As will be proved later, the sum of the coefficients of $R_{n}$ is the $n$th Motzkin number, and the sum of the coefficients of $R_{2 n}^{\prime}$ is the $n$th Catalan number. An immediate consequence of Theorem 1 is:

Corollary 2. The polynomials $R_{n}$ and $R_{n}^{\prime}$ have the expression

$$
R_{n}(X) \equiv \operatorname{char}\left(\mathscr{P} \cap X^{n}\right), \quad R_{n}^{\prime}(X) \equiv \operatorname{char}\left(\mathscr{P} \cap X^{\prime^{n}}\right),
$$

where $a \equiv b$ means that $a$ and $b$ are equivalent modulo the commutativity of the indeterminates $X$.

This interpretation compares to Touchard's remarks concerning the $R_{n}^{\prime}$. In our case, we mark both rises and falls (by $a$ 's and $b$ 's respectively); Touchard's interpretation corresponds to the case where all $b_{j}$ are set to 1 , i.e. falls are unmarked. Indeed if we let $X_{1}^{\prime}=\sigma_{1}\left(X^{\prime}\right)$ where $\sigma_{1}\left(b_{j}\right)=1$ for all $j \geqslant 0$, we have

$$
R_{n}^{\prime}\left(X_{1}^{\prime}\right)=\sum_{i_{1}=0} \sum_{0 \leqslant i_{2} \leqslant i_{1}+1} \sum_{0 \leqslant i_{3} \leqslant i_{2}+1} \cdots \sum_{0 \leqslant i_{n} \leqslant i_{n-1}+1} a_{i_{1}} a_{i_{2}} \cdots a_{i_{n}},
$$

which is precisely Touchard's expression of the Stieltjes-Rogers polynomials.

The Jacobi-Rogers polynomials $R_{n}$ are homogeneous polynomials. As a consequence of Corollary 2, we now show that their coefficients have closed form expressions.

Proposition 3A. The Jacobi-Rogers polynomials have the explicit expression:

$$
R_{n}(X)=\sum_{\substack{h, n_{0}, \ldots, n_{h} ; \\ m_{0}, \ldots, m_{h}}} \rho\left(n_{0}, \ldots, n_{h} ; m_{0}, \ldots, m_{h}\right)\left(a_{0} b_{1}\right)^{n_{0}} \cdots\left(a_{h} b_{h+1}\right)^{n_{h}} c_{h}^{m_{h}}
$$


where the sum ranges over all $h \geqslant 0$ and all sequences $\left(n_{0}, \ldots, n_{h} ; m_{0}, \ldots, m_{h}\right)$ such that $2 n_{0}+2 n_{1}+\cdots+2 n_{h}+m_{0}+m_{1}+\cdots+m_{h}=n$, and $\rho$ is given by

$$
\begin{aligned}
& \rho\left(n_{0}, \ldots, n_{h} ; m_{0}, \ldots, m_{h}\right) \\
& =\left(\begin{array}{c}
n \\
m_{0}
\end{array}\right)\left(\begin{array}{c}
n_{0}+n_{1}-1 \\
n_{0}-1
\end{array}\right)\left(\begin{array}{c}
n-m_{0}-2 n_{1} \\
m_{1}
\end{array}\right)\left(\begin{array}{c}
n_{1}+n_{2}-1 \\
n_{1}-1
\end{array}\right) \cdots \\
& \quad \ldots\left(\begin{array}{c}
n_{h-1^{-}}+n_{h-1} \\
n_{h-1}-1
\end{array}\right)\left(\begin{array}{c}
n-m_{0}-\cdots-m_{h-1}-2 n_{0} \cdots-2 n_{h-1} \\
m_{h}
\end{array}\right) .
\end{aligned}
$$

Conventionally $\left(\stackrel{p}{1}_{1}^{\mathbf{p}}\right),=\delta_{p,-1}$ where $\delta$ is Kronecker's symbol.

Proof. The binomials

$$
\left(\begin{array}{c}
n \\
m_{0}
\end{array}\right), \quad\left(\begin{array}{c}
n-m_{0}-2 n_{0} \\
m_{1}
\end{array}\right), \ldots
$$

count the number of ways of inserting level steps at height $0,1, \ldots$ The binomials

$$
\left(\begin{array}{c}
n_{r}+n_{r+1}-1 \\
n_{r}-1
\end{array}\right)
$$

count the number of ways of associating $n_{r}$ points at level $r$ to $n_{r+1}$ points at level $r+1$, in a way consistant with the rules defining paths.

In the case of the Stieltjes-Rogers polynomials, the expression assumes a nicer form:

Proposition 3B. The Stieltjes-Rogers polynomials have the explicit expression:

$$
\begin{aligned}
R_{2 n}^{\prime}\left(X^{\prime}\right)= & \sum_{\substack{h \geqslant 0 \\
n_{0}+\cdots+n_{h}=n}}\left(\begin{array}{c}
n_{0}+n_{1}-1 \\
n_{0}-1
\end{array}\right)\left(\begin{array}{c}
n_{1}+n_{2}-1 \\
n_{1}-1
\end{array}\right) \cdots\left(\begin{array}{c}
n_{h-1}+n_{h}-1 \\
n_{h-1}-1
\end{array}\right) \\
& \times\left(a_{0} b_{1}\right)^{n_{0}}\left(a_{1} b_{2}\right)^{n_{1}} \cdots\left(a_{h} b_{h+1}\right)^{n_{h}} .
\end{aligned}
$$

The $k$ th convergents $J^{[k]}$ and $S^{[k]}$ have similar expansions in which the index $h$ in the summation is restricted to the range $0, \ldots, \mathrm{k}-1$.

\subsection{Continued fractions and power series: the Stieltjes matrix}

As shown by Stieltjes [26], the relations between continued fractions and power series can be described in terms of matrix equations. We show here that the elements of the Stieltjes matrix also have simple combinatorial interpretations.

We first recall Stieltjes's theorem in the form given by Wall [30, p. 203].

Theorem S. (Stieltjes's expansion theorem for $J$-fractions). The coefficients in the $J$-fraction

$$
\frac{1}{1-c_{0} z-\frac{a_{0} z^{2}}{1-c_{1} z-\frac{a_{1} z^{2}}{\cdots}}}
$$


and its power series expansion $D(z)=\sum_{n \geqslant 0} d_{n} z^{n}$ are connected by the relations $d_{p}=k_{0, p}$ for all $p \geqslant 0$ and more generally

$$
d_{p+q}=k_{0, p}+a_{1} k_{1, p} k_{1, q}+a_{1} a_{2} k_{2, p} k_{2, q}+\cdots \text { for all } p, q \geqslant 0
$$

where $k_{0,0}=1, k_{r, s}=0$ if $r>s$, and the $k_{r, s}$ satisfy the matrix equation:

$$
\begin{aligned}
&\left(\begin{array}{lllll}
k_{01} & k_{11} & 0 & 0 & \cdots \\
k_{02} & k_{12} & k_{22} & 0 & \ldots \\
k_{03} & k_{13} & k_{23} & k_{33} & \ldots
\end{array}\right)= \\
&\left(\begin{array}{cccccc}
k_{00} & 0 & 0 & 0 & \cdots \\
k_{01} & k_{11} & 0 & 0 & \cdots \\
k_{02} & k_{12} & k_{22} & 0 & \ldots
\end{array}\right) \cdot\left(\begin{array}{ccccc}
c_{0} & 1 & 0 & 0 & \cdots \\
a_{0} & c_{1} & 1 & 0 & \ldots \\
0 & a_{1} & c_{2} & 1 & \ldots
\end{array}\right) .
\end{aligned}
$$

Classically, a progressive construction of the Stieltjes matrix $\left(k_{r, s}\right)$ is used as an easy way of expanding the $J$-fraction into power series; conversely recurrent determination of the Stieltjes matrix from its first column leads to an efficient way for computing the coefficients of the corresponding $J$-fraction $[26,23,19,30]$.

We now prove a non-commutative analogue of Stieltjes' theorem. First define for all $k, l \geqslant 0$ the sets

$$
\mathscr{P}_{k, l}=\left\{u \in X^{*} \mid a_{0} a_{1} \cdots a_{k-1} u s_{l} s_{l-1} \cdots s_{1} \in \mathscr{P}\right\} .
$$

The elements of $\mathscr{P}_{k, l}$ will be sometimes referred to as extended paths from (height) $k$ to (height) $l$, and we have $\mathscr{P}=\mathscr{P}_{0,0}$.

Proposition 4. In the set of infinite matrices over $C\langle X X\rangle$, the following equality holds:

$$
\Pi=T \Pi \Delta+I
$$

where

$$
\begin{aligned}
(T)_{i j} & =\delta_{i-1, j} ; \quad(I)_{i j}=\delta_{i 0} \delta_{j 0} \\
\Delta & =\left(\begin{array}{ccccc}
c_{0} & a_{0} & 0 & 0 & \ldots \\
b_{1} & c_{1} & a_{1} & 0 & \ldots \\
0 & b_{2} & c_{2} & a_{2} & \ldots
\end{array}\right)
\end{aligned}
$$

and $\Pi$ is a matrix of extended paths:

$$
(\Pi)_{i j}=\operatorname{char}\left(\mathscr{P}_{0 j} \cap X^{i}\right) \text {. }
$$

In particular the sum of the first column of $\Pi$ is equal to $\operatorname{char}(\mathscr{P})$.

Proof. The proof is the matrix translation of the obvious recurrences:

$$
\mathscr{P}_{0, j} \cap X^{i+1}=\left(\mathscr{P}_{0, j-1} \cap X^{i}\right) a_{j}+\left(\mathscr{P}_{0, j} \cap X^{i}\right) c_{j}+\left(\mathscr{P}_{0, j+1} \cap X^{i}\right) b_{j+1} \text {, }
$$

for $j \geqslant 1$ and $i \geqslant 0$. 
Example. Writing explicitly the first elements of $\Pi, T, \Delta, I$, the identity expressed in Proposition 4 reads:

$$
\begin{gathered}
\left(\begin{array}{cccc}
1 & 1 & 0 & \cdots \\
c_{0} & a_{0} & 0 & \cdots \\
c_{0}^{2}+a_{0} b_{1} & c_{0} a_{0}+a_{0} c_{1} & a_{0} a_{1} & \cdots
\end{array}\right)=\left(\begin{array}{cccc}
0 & 0 & 0 & \cdots \\
1 & 0 & 0 & \cdots \\
0 & 1 & 0 & \cdots \\
\cdots & \cdots
\end{array}\right) \\
\times\left(\begin{array}{ccc}
1 & 0 & \cdots \\
c_{0} & a_{0} & \cdots \\
& \cdots &
\end{array}\right)\left(\begin{array}{ccccc}
c_{0} & a_{0} & 0 & 0 & \cdots \\
b_{1} & c_{1} & a_{1} & 0 & \cdots \\
0 & b_{2} & c_{2} & a_{2} & \cdots
\end{array}\right)+\left(\begin{array}{cccc}
1 & 0 & 0 & \cdots \\
0 & 0 & 0 & \cdots \\
0 & 0 & 0 & \cdots \\
& & \cdots &
\end{array}\right) .
\end{gathered}
$$

The elements on the diagonal of $M$ are $1, a_{0}, a_{0} a_{1}, a_{0} a_{1} a_{2}, \ldots$ and the elements immediately below are $c_{0}, c_{0} a_{0}+a_{0} c_{1}, c_{0} a_{0} a_{1}+a_{0} a_{1} c_{2}, \ldots$

Proposition 4 trivially entails Theorem S. The proof here is achieved without determinant manipulations and it reveals a simple combinatorial interpretation of the Stieltjes matrix. As noticed by Rogers [23, 30, p. 204, 19], Theorem S can be interpreted as an addition formula: if in the notations of Theorem $\mathrm{S}$ we let

$$
f(x)=\sum_{n \geqslant 0} k_{0, n} \frac{x^{n}}{n !} \text { and } f_{r}(x)=\sum_{n \geqslant r} k_{r, n} \frac{x^{n}}{n !},
$$

then

$$
f(x+y)=f_{0}(x) f_{0}(y)+a_{0} f_{1}(x) f_{1}(y)+a_{0} a_{1} f_{2}(x) f_{2}(y)+\cdots .
$$

We shall see in Section 3, that allowing commutativity leads to a simple combinatorial expression for the matrix $\Pi^{-1}$.

\section{Enumerative properties of continued fractions}

The equivalence between paths and continued fractions leads to direct continued fraction expressions of generating functions relative to path enumerations. The introduction of various classes of path diagrammes which are in natural correspondance with set partitions and permutations shows the process to apply in these cases also. Finally the observations of Section 1 lead to a simple treatment of Carlitz's cycles of binomial coefficients [2].

It should be emphasized here that continued fractions are introduced without any computation over generating functions, by simply observing direct geometrical correspondances between objects. The steps we take here makes it possible to read off the properties of the enumerated objects directly on the continued fraction. As explained in the introduction this attitude towards enumeration problems originates in the works of Schützenberger and Foata (see especially [11]). 


\subsection{Paths in the plane}

Let $M_{n}$ be the number of paths of length $n$, and let $C_{n}$ be the number of paths of length $2 n$ without level steps:

$$
M_{n}=\operatorname{card}\left(\mathscr{P} \cap X^{n}\right) ; \quad C_{n}=\operatorname{card}\left(\mathscr{P} \cap X^{\prime 2 n}\right) .
$$

The $M_{n}$ are the Motzkin numbers, and the $C_{n}$ the Catalan numbers: $\left\{M_{n}\right\}=$ $\{1,1,2,4,9,21 \ldots\}$ and $\left\{C_{n}\right\}=\{1,1,2,5,14,42 \ldots\}$. Using the morphism $\mu$ : $C\langle\langle X\rangle\rangle \rightarrow C[[z]]$ defined by $\mu\left(a_{j}\right)=\mu\left(c_{j}\right)=z$ for all $j \geqslant 0$, and $\mu\left(b_{j}\right)=z$ for all $j \geqslant 1$ i.e. counting all steps by $z$ we get as immediate application of Theorem 1:

Proposition 5. The generating function of the Motzkin and Catalan numbers have the expansions:

$$
\begin{aligned}
& \sum_{n \geqslant 0} M_{n} z^{n}=\frac{1}{1-z-\frac{z^{2}}{1-z-\frac{z^{2}}{\cdots}}} ; \\
& \sum_{n \geqslant 0} C_{n} z^{2 n}=\frac{1}{1-\frac{z^{2}}{1-\frac{z^{2}}{\cdots}}} .
\end{aligned}
$$

As is well known the generating functions have the expressions

$$
\sum_{n \geqslant 0} M_{n} z^{n}=\frac{1+z-\sqrt{1-2 z+3 z^{2}}}{2 z} ; \quad \sum_{n \geqslant 0} C_{n} z^{n}=\frac{1-\sqrt{1-4 z}}{2 z} .
$$

Classically, Proposition 5 is derived as a consequence of the (periodic) continued fraction expansion for a quadratic irrationality. The expansion of the Catalan series in the context of enumeration problems arises in $[28,1]$.

By utilizing various morphisms, we can derive expressions for other generating functions. Let for instance $M_{n, k}$ denote the number of paths in $\mathscr{P}$, of length $n$ containing $k$ level steps. The generating function $\sum M_{n, k} u^{k} z^{n}$ is obtained by means of the morphism:

$$
\mu\left(a_{j}\right)=\mu\left(b_{k}\right)=z ; \quad \mu\left(c_{j}\right)=z u \text { for all } j \geqslant 0, k \geqslant 1 .
$$

So that:

$$
\sum_{n, k \geqslant 0} M_{n, k} u^{k} z^{n}=\frac{1}{1-z u-\frac{z^{2}}{1-z u-\frac{z^{2}}{\cdots}}} .
$$

On the other hand, as is not difficult to see

$$
\sum_{n, k \geqslant 0} M_{n, k} u^{k} z^{n}=\frac{1+(2-u) a-\sqrt{(1-u z)^{2}-4 z^{2}}}{2 z} .
$$

In a very similar way, we can derive an expansion of the generating function of binary trees according to number of leaves (terminology is the same as in [15]). 
We now turn to a different type of enumeration result related to the so-called $q$-generations. Define the area below a path without level steps as the sum of the height of the points in the path. The area $\alpha(u)$ of path $u$ is defined inductively by: $\alpha(\varepsilon)=0 ; \alpha\left(a_{j}\right)=j ; a\left(b_{k}\right)=k$ for all $j \geqslant 0, k \geqslant 1$; and $\alpha(x y)=\alpha(x)+\alpha(y)$ for $x, y$ in $X^{*}$.

The area is always an odd integer. Let $A_{n, k}$ denote the number of paths of length $n$ having area $k$. The morphism $\mu$ is defined by

$$
\mu\left(a_{j}\right)=z q^{j} \quad \text { and } \quad \mu\left(b_{k}\right)=z q^{k} \quad \text { for all } j \geqslant 0, k \geqslant 1,
$$

so that $\mu(u)=z^{|u|} q^{\alpha(u)}$. Applying it to the equality given by Theorem 1 , yields the following result first obtained by Carlitz [3]:

Proposition 6. The generating function of paths partitioned according to area has the expansion

$$
A(z, q)=\sum A_{n, k} z^{n} q^{k}=\frac{1}{1-\frac{z^{2} q^{1}}{1-\frac{z^{2} q^{3}}{1-\frac{z^{2} q^{5}}{\cdots}}}} .
$$

This continued fraction has been studied by Ramanujan (see [19, p. 126]), who showed it to be expressible in terms of the $q$-exponential function:

$$
G(x)=1+\sum_{n \geqslant 1} \frac{q^{n^{2}} x^{n}}{(1-q)\left(1-q^{2}\right) \cdots\left(1-q^{n}\right)} .
$$

\subsection{Path diagrammes and set partitions}

Path diagrammes are related to objects considered by J. Rosen under the name of weighted lead ballot sequences [24]. They also appear in various forms in works by Strehl [27] and Dumont [7]. However their systematic use in enumeration problems relative to permutations is due to Françon and Viennot $[12,13]$.

Definition. A system of path diagrammes is defined by an application pos: $X \rightarrow N$ called a possibility function; a path diagramme is a couple $(u, s)$ where $u=$ $u_{1} u_{2} \cdots u_{n}$ is a path and $s$ is a sequence of integers $s=s_{1} s_{2} \cdots s_{n}$ such that for all $j 0 \leqslant s_{j}<\operatorname{pos}\left(u_{j}\right)$.

A path diagramme $(u, s)$ where $|u|=n$ can be represented by a path together with a set of $n$ points $\left.\left.\left.P_{1}\right|_{y_{1}} ^{0} P_{2}\right|_{y_{2}} ^{1} \cdots P_{n}\right|_{y_{n}} ^{n-1}$ where the abscissae $y_{j}$ are integers subjected to the condition $0 \leqslant y_{j}<\operatorname{pos}\left(u_{j}\right)$.

Example. Consider the system defined by the possibility function:

$$
\operatorname{pos}\left(a_{j}\right)=\operatorname{pos}\left(c_{j}\right)=j+1 \text { and } \operatorname{pos}\left(b_{k}\right)=k+1 \text { for all } j \geqslant 0, k \geqslant 1 \text {. }
$$


A diagramme belonging to this system is

$$
d=(u ; s)=\left(a_{0} a_{1} c_{2} b_{2} a_{1} a_{2} b_{3} b_{2} c_{1} b_{0} ; 0,1,0,2,0,1,3,1,0,0\right) ;
$$

it can graphically represented as in Fig. 2 with the $P_{j}$ 's being drawn as crosses.

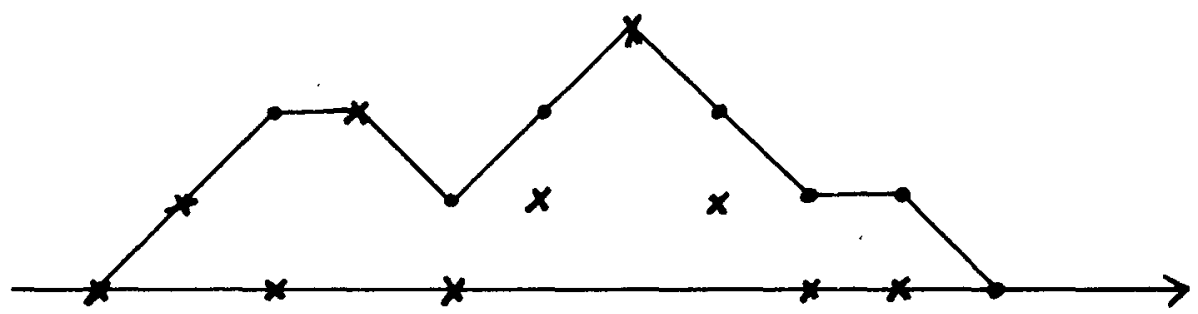

Fig. 2

Path diagrammes can also be described as words over a labelled alphabet

$$
Y=\left\{a_{i}^{(j)}, b_{k}^{(j)}, c_{i}^{(j)}\right\}_{i, j \geqslant 0} \quad k \geqslant 1
$$

where letter $x_{i}^{(j)}$ represents the $j$ th possibility relative to $x_{1} \in X$. Thus for the diagramme in the last example the representation (also denoted $d$ ) is

$$
d=\left(a_{0}^{(0)}, a_{1}^{(1)}, c_{2}^{(0)}, b_{2}^{(2)}, a_{1}^{(0)}, b_{3}^{(3)}, b_{2}^{(1)}, c_{1}^{(0)}, b_{0}^{(0)}\right) .
$$

Let $\mathscr{D}$ be the set of path diagrammes relative to the possibility function pos; path diagrammes are obtained from path by substituting to each variable $\omega_{j}(\omega=$ $a, b$ or $c$ ) the sum of all corresponding possibilities. Thus:

Proposition 7A. The non-commutative series char $(\mathscr{D})$ has the non-commutative continued fraction expansion:

$$
\operatorname{char}(\mathscr{D})=\frac{1}{1-\left(c_{0}^{(0)}+\cdots+c_{0}^{(\gamma)}\right)-\frac{\left(a_{0}^{(0)}+\cdots+a_{0}^{(\alpha)}\right) \mid\left(b_{1}^{(0)}+\cdots+b_{1}^{(\beta)}\right)}{1-\left(c_{1}^{(0)}+\cdots+c_{1}^{\left(\gamma^{\prime}\right)}\right)-\frac{\left(a_{1}^{(0)}+\cdots+a_{1}^{\left(\alpha^{\prime}\right)}\right) \mid\left(b_{2}^{(0)}+\cdots+b_{2}^{\left(\beta^{\prime}\right)}\right)}{\cdots}}}
$$

where $1+\gamma=\operatorname{pos}\left(c_{0}\right) ; 1+\alpha=\operatorname{pos}\left(a_{0}\right) ; 1+\beta=\operatorname{pos}\left(b_{1}\right) ; 1+\gamma^{\prime}=\operatorname{pos}\left(c_{1}\right)$, etc.

In the sequel, we shall freely extend to path diagrammes the terminology relative to paths: we shall thus speak of the length and height of a diagramme; we shall consider diagrammes without level steps....

The importance of path diagrammes in the context of enumeration problems comes from the following:

Proposition 7B. Let $D_{n}$ denote the number of path diagrammes of length $n$ relative to a possibility function:

$$
\operatorname{pos}\left(a_{j}\right)=\alpha_{j} ; \quad \operatorname{pos}\left(b_{k}\right)=\beta_{k} ; \quad \operatorname{pos}\left(c_{j}\right)=\gamma_{j} \quad \text { for } j \geqslant 0, k \geqslant 1 .
$$


The generating function $D(z)=\sum_{n \geqslant 0} D_{n} z^{n}$ has the expansion:

$$
D(z)=\frac{1}{1-\gamma_{0} z-\frac{\alpha_{0} \beta_{1} z^{2}}{1-\gamma_{1} z-\frac{\alpha_{1} \beta_{2} z^{2}}{1-\gamma_{2} z-\frac{\alpha_{2} \beta_{3} z^{2}}{\cdots}}}} .
$$

Proof. It immediately follows from Proposition 7A, using the morphism $\mu$ defined by $\mu\left(\omega_{j}^{(i)}\right)=z$ for $\omega \in\{a, b, c\}$ and $i, j \in \mathbf{N}$.

Path diagrammes are of interest as they can be put into simple correspondence with many usual combinatorial objects like permutations, set partitions .... They thus appear as the adequate tool for obtaining continued fraction expansions of ordinary generating functions. Notice that any $J$-fraction with integral coefficients enumerates a certain system of path diagrammes for which interpretations can be sought (see Sections 2-3).

We now exhibit a correspondence between a system of path diagrammes and set partitions. This correspondence extends some previous results by Françon and Viennot [13] relative to involutions, i.e. to partitions into singletons and doubletons only. We have:

Proposition 8. Set partitions of size $n$ are in one-to-one correspondence with diagrammes of length $n$ relative to the possibility function defined by

$$
\operatorname{pos}\left(a_{j}\right)=1 ; \quad \operatorname{pos}\left(b_{k}\right)=k+1 ; \quad \operatorname{pos}\left(c_{j}\right)=j+1 \quad \text { for all } j \geqslant 0, k \geqslant 1 \text {. }
$$

Proof. The proof is constructive. We start with a partition $\pi$ relative to a set of $n$ elements which we assume to be canonically numbered $\{1,2, \ldots, n\}$, and we construct a path diagramme $(u, s)$ of length $n$. Given $\pi$, elements of $[1 \cdots n]$ are divided into three classes:

(1) opening elements: these are elements belonging to a class of cardinality $\geqslant 2$ which are smallest in their class;

(2) closing elements: these are elements belonging to a class of cardinality $\geqslant 2$ which are largest in their class;

(3) transient elements: all other elements, i.e. either non extremal elements of classes of cardinality $\geqslant 2$, or elements of singleton classes.

Let $v=v_{1} v_{2} \cdots v_{n}$ be the unlabelled path corresponding to $u$; the $v_{j}$ 's are defined by

$v_{j}=a$ if $j$ is an opening element in $r$,

$v_{j}=c$ if $j$ is a closing element in $r$,

$v_{j}=. b$ if $j$ is a transient element in $r$.

Now the sequence $s_{1} s_{2} \cdots s_{n}$ is constructed as follows:

(a) if $j$ is an opening element (equivalently if $v_{j}=a$ ), then $s_{j}=0$. Giving an 
element $j$ and a class $\alpha=\left\{\alpha_{1} \leqslant \alpha_{1} \leqslant \cdots \leqslant \alpha_{s}\right\}$, we say that $\alpha$ overlaps with $j$ iff $\alpha_{1}<j \leqslant \alpha_{s}$; then:

(b) if $j$ is a closing element or a transient element in a class of cardinality $\geqslant 2$, we consider the classes ovelapping $j$ and we arrange them according to the order of their first elements. Let $\left\{\alpha_{01} \leqslant \alpha_{02} \leqslant \cdots \leqslant \alpha_{0 s_{0}}\right\},\left\{\alpha_{11} \leqslant \alpha_{12} \leqslant \cdots \alpha_{1 s_{1}}\right\}, \cdots$ be these overlapping classes with $\alpha_{01}<\alpha_{11}<\alpha_{21}<\cdots$. If $j$ belongs to the class $\left\{\alpha_{\nu, 1} \leqslant \alpha_{\nu, 2} \leqslant \cdots\right\}$, then we set $s_{j}=\nu$.

(c) if $j$ belongs to a singleton class, $s_{j}$ is equal to the number of classes overlapping $j$.

The correspondence is readily checked to yield a path diagramme consistent with the possibility rules:

$$
\operatorname{pos}\left(a_{j}\right)=1 ; \quad \operatorname{pos}\left(b_{k}\right)=k+1 ; \quad \operatorname{pos}\left(c_{j}\right)=j+1,
$$

and it is obviously revertible.

Example. The somewhat esoteric nature of this correspondence is easily uncovered by an example. Take for instance $n=13$, and consider the partition

$$
\pi=\{1,7,11\}\{2,4,6,9\}\{3\}\{5,10\}\{8\}\{12,13\} .
$$

The partition can be graphically represented in a simple way (see Fig. 3).

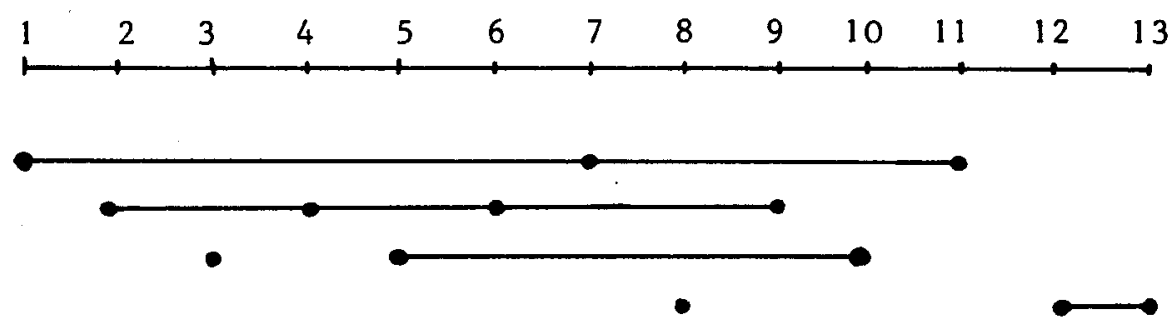

Fig. 3

On this graph, we see that $\{1,7,11\}\{2,4,6,9\}$ and $\{5,10\}$ overlap with the element 6 . The unlabelled path of the diagramme associated to $\pi$ is $v=$ $v_{1} v_{2} \cdots v_{13}$. The element 1 opens class $\{1,7,11\}$ so: $v_{1}=a$; the element 2 opens class $\{2,4,6,9\}$, so $v_{2}=a$; the element 3 is transient (member of a singleton class), so $v_{3}=c ; 4$ is also transient so $v_{4}=c, \ldots$, until $v_{13}$ which is a $b$ since 13 closes the class $\{12,13\}$. Thus we have

$$
v=a a c c a c c c b b b a b \text { and } u=a_{0} a_{1} c_{2} c_{2} a_{2} c_{3} c_{3} c_{3} b_{3} b_{2} b_{1} a_{0} b_{1} .
$$

Now the sequence $s_{1} s_{2} \cdots s_{13}$ is also easily determined: $s_{1}=s_{2}=0$ since 1 and 2 are opening elements. At point 3 , which is a singleton class, two classes are opened; these are $\{1 \cdots\}$ and $\{2 \cdots\}$, so that we take $s_{3}=2$. Element 4 is transient in the second opened class, so that $s_{4}=1$ (we rank classes starting from zero!). Ultimately we have $s=0021010311000$, and the planar representation of the diagramme $(u, s)$ is shown in Fig. 4. 


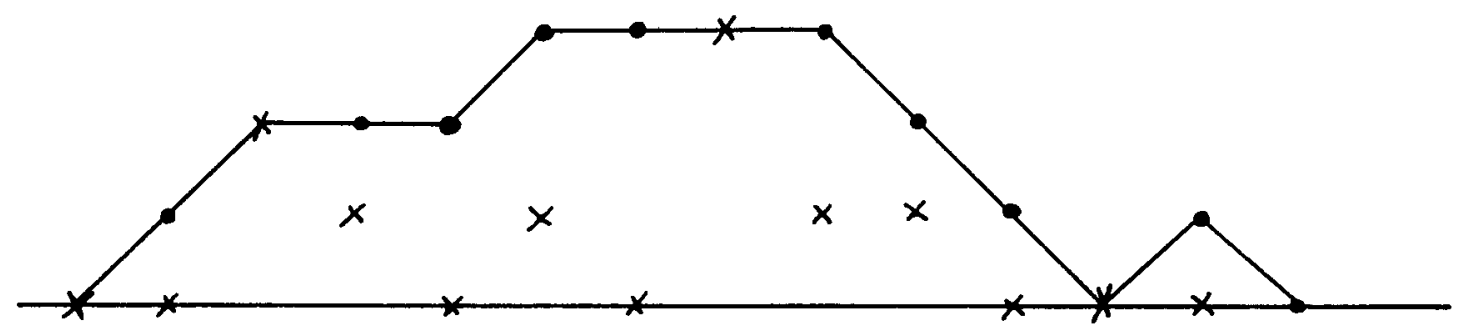

Fig. 4

Using this correspondence, we get:

Theorem 2. Let $\beta_{n_{1}, n_{2}, m}$ be the number of partitions having $n_{1}$ singleton classes, $n_{2}$ classes of cardinality $\geqslant 2$, and $m$ non-singleton transient elements, then the generating function

$$
\beta\left(u_{1}, u_{2}, t, z\right)=\sum_{n_{1}, n_{2}, m \geqslant 0} \beta_{n_{1}, n_{2}, m} u_{1}^{n_{1}} u_{2}^{n_{2}} t^{m} z^{m+n_{1}+2 n_{z}}
$$

has the expansion:

$$
\beta\left(u_{1}, u_{2}, t, z\right)=\frac{1}{1-u_{1} z-\frac{1 u_{2} z^{2}}{1-\left(u_{1}+1 t\right) z-\frac{\left.1 u_{1}+2 t\right) z-\frac{3 u_{2} z^{2}}{\cdots}}{1-\left(u_{2}\right.}} 2 u_{2} z^{2}} .
$$

In particular:

$$
\text { (ia) } \sum B_{n} z^{n}=\frac{1}{1-1 z-\frac{1 z^{2}}{1-2 z-\frac{2 z^{2}}{\cdots}}}
$$$$
\text { (iia) } \sum I_{n} z^{n}=\frac{1}{1-z-\frac{1 z^{2}}{1-z-\frac{2 z^{2}}{\cdots}}}
$$

(ib) $\sum S(n, k) u^{k} z^{n}=\frac{1}{1-u z-\frac{1 u z^{2}}{1-(1+u) z-\frac{2 u z^{2}}{\cdots}}}$

$$
\text { (iib) } \sum J_{n} z^{n}=\frac{1}{1-\frac{1 z^{2}}{1-\frac{2 z^{2}}{1-\frac{3 z^{2}}{\cdots}}}}
$$

where the $B_{n}$ are Bell's exponential numbers; the $S(n, k)$ are the Stirling numbers of the first kind; $I_{n}$ counts involutions on $n ; J_{n}$ counts involutions on $n$ having no fixed point, i.e. $J_{2 n}=1 \cdot 3 \cdot 5 \cdots(2 n-1) ; J_{2 n+1}=0$.

Proof. We use the morphism:

$$
\mu\left(a_{j}\right)=u_{2} z ; \quad \mu\left(b_{k}\right)=k z ; \quad \mu\left(c_{j}\right)=\left(u_{1}+(j-1) t\right) z, \quad \text { for } j \geqslant 0, k \geqslant 1 .
$$

The other cases are special applications, for instance (ia) is derived by setting $u_{1}=u_{2}=t=1$, (iia) by $u_{1}=u_{2}=1, t=0$. 
Similar expressions hold for the (2-) associated Bell and Stirling numbers counting partitions without singleton classes [5, vol 2 p. 57]. Call $B_{n}^{(2)}$ the number of such partitions of a set of $n$ elements, and $S_{n, k}^{(2)}$ the number of those comprising $k$ classes:

$$
\begin{aligned}
\sum_{n \geqslant 0} B_{n}^{(2)} z^{n} & =\frac{1}{1-\frac{1 z^{2}}{1-1 z-\frac{2 z^{2}}{1-2 z-\frac{3 z^{2}}{\cdots}}}} \\
\sum_{n, k \geqslant 0} S_{n}^{(2)} z^{n} & =\frac{1}{1-\frac{1 u z^{2}}{1-1 z-\frac{2 u z^{2}}{\cdots}}}
\end{aligned}
$$

Notice that all these quantities have exponential generating functions of a simple form;

$$
\beta\left(u_{1}, u_{2}, t, z\right)=\sum \beta_{n_{1}, n_{2}, m} u_{1}^{n_{1}} u_{2}^{n_{2}} t^{m} \frac{z^{n_{1}+m+2 n_{2}}}{\left(n_{1}+m+2 n_{2}\right) !}
$$

is given by:

$$
\beta\left(u_{1}, u_{2}, t, z\right)=\exp \left(u_{1} z+u_{2}\left(\frac{z^{2}}{2 !}+\frac{z^{3} t}{3 !}+\frac{z^{4} t^{2}}{4 !}+\cdots\right)\right) .
$$

In particular as is well known:

$$
\begin{aligned}
& \sum B_{n} \frac{z^{n}}{n !}=\exp \left(\mathrm{e}^{z}-1\right) \\
& \sum S(n, k) u^{k} \frac{z^{n}}{n !}=\exp u\left(\mathrm{e}^{z}-1\right) ; \sum I_{n} \frac{z^{n}}{n !}=\mathrm{e}^{z+z^{2} / 2} ; \quad \sum J_{n} \frac{z^{n}}{n !}=\mathrm{e}^{z^{2} / 2} \\
& \sum B_{n}^{(2)} \frac{z^{n}}{n !}=\exp \left(\mathrm{e}^{z}-z-1\right) ;
\end{aligned}
$$

and

$$
\sum S_{n, k}^{(2)} u^{k} \frac{z^{n}}{n !}=\exp u\left(\mathrm{e}^{z}-z-1\right) .
$$

It is possible to use a formal Laplace-Borel-transform $L(f(t) ; u)$ defined by $L\left(t^{k} ; u\right)=k ! u^{k}$ and corresponding to the analytical expression

$$
L(f(t) ; u)=\int_{0}^{\infty} e^{-t} f(t u) \mathrm{d} t
$$

to rephrase Theorem 2 as continued fraction expansions of the Laplace transforms of some exponential series.

The continued fraction relative to the Bell numbers is implicit in several 
analytic works relative to the Charlier polynomials [4]. The continued fraction relative to the odd factorial numbers $J_{n}$ is classically derived as a limiting case of the continued fraction of Gauss which expresses the quotient of two contiguous hypergeometric functions.

\subsection{Permutations}

In this section, we use a fundamental bijection due to Françon and Viennot $[13,12]$ between a certain system of path diagrammes and permutations, to derive continued fraction expansions relative to the factorial, Euler and Eulerian numbers. We then use. Theorem 1 together with a classical expansion to construct a new interpretation of the coefficients of the elliptic functions $\mathrm{cn}, d n$ which is not trivially reducible to the first known interpretation due to Viennot [29].

In order to better understand the correspondence between permutations and path diagrammes, we first recall the representation of permutations by tournament trees. A tournament tree is a binary tree with node labels that increase along each branch. Given a permutation $\sigma=\sigma_{1} \sigma_{2} \cdots \sigma_{n}$ of $[1 \cdots n]$, such that 1 occurs at position $i$, i.e. $\sigma=\sigma_{1} \sigma_{2} \cdots \sigma_{i-1} 1 \sigma_{i+1} \cdots \sigma_{n}$, the tournament tree associated to $\sigma$ is obtained by putting 1 at the root and by taking as left subtree the tournament tree recursively associated to $\sigma_{1} \cdots \sigma_{i-1}$, and by taking as right subtree the tournament tree recursively associated to $\sigma_{i+1} \cdots \sigma_{n}$.

For instance to the permutation $\sigma=(1,7,10,4,8,6,9,2,5,3)$ there corresponds the tree shown in Fig. 5.

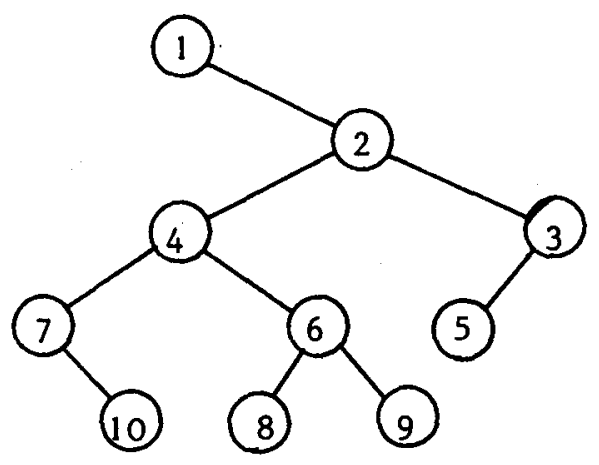

Fig. 5

Conversely reading the labels of the tree in left to right order gives back the original permutation.

We now state Françon and Viennot's theorem [12]:

Theorem F-V. (The fundamental correspondence between path diagrammes and permutations). Permutations of $[n+1]$ are in one-to-one correspondence with path diagrammes corresponding to the possibility function

$$
\operatorname{pos}\left(a_{j}\right)=j+1 ; \quad \operatorname{pos}\left(b_{k}\right)=k+1 ; \quad \operatorname{pos}\left(c_{j}\right)=2 j+2 \quad \text { for all } j \geqslant 0, k \geqslant 1 .
$$

Proof. We only sketch the proof here, referring the reader to [12] for a precise description of the correspondence. 
For ease of presentation, we shall set $c_{j}=c_{j}^{\prime}+c_{j}^{\prime \prime}$. The characteristic series for paths char $(\mathscr{P})$ then becomes a series on the alphabet

$$
X^{\prime \prime}=\left\{a_{j}\right\}_{j \geqslant 0} \cup\left\{b_{k}\right\}_{k \geqslant 1} \cup\left\{c_{j}^{\prime}\right\}_{j \geqslant 0} \cup\left\{c_{j}^{\prime \prime}\right\}_{j \geqslant 0} \text {. }
$$

In other words level steps can be marked either by a prime (') or by a double prime ("). Path diagrammes relative to alphabet $X^{\prime \prime}$ can be defined in a similar way, and we readily check that the path diagrammes corresponding to

$$
\operatorname{pos}\left(a_{j}\right)=j+1 ; \quad \operatorname{pos}\left(b_{k}\right)=k+1 ; \quad \operatorname{pos}\left(c_{j}\right)=2 j+2,
$$

are in correspondence with path diagrammes defined by

$$
\operatorname{pos}\left(a_{j}\right)=j+1 ; \quad \operatorname{pos}\left(b_{k}\right)=k+1 ; \quad \operatorname{pos}\left(c_{j}^{\prime}\right)=j+1 ; \quad \operatorname{pos}\left(c_{j}^{\prime \prime}\right)=j+1 .
$$

Now starting with a diagramme $(v, t)$ of length $n$ we describe the algorithm that constructs a tournament tree over $[n+1]$. Our terminology concerning binary trees is again that of Knuth [15].

The algorithm proceeds by successive insertion of nodes $1,2,3, \ldots$ starting from an empty tree at stage 0 which corresponds to one position to be filled. At stage $j$ for $1 \leqslant j \leqslant n, j$ positions are available to insert node $j$. If the letter $v_{j}$ is an $a$, the node labelled by $j$ is taken to be a double node; if $v_{j}$ is a $b$, the node $j$ is a leaf; if $v_{j}$ is a $c^{\prime}, j$ is a left branching node; finally if $v_{j}$ is a $c^{\prime \prime}, j$ is a right branching node.

At each stage $j$, when the height in the path is $h_{j}$, the number of vacant links is $1+h_{j}$ before $j$ is inserted. If the number in the possibility sequence is $s_{j}$, we assign node $j$ at the $1+s_{j}$ vacant position starting from the left.

The construction is terminated by putting node $(n+1)$ as a leaf in the last vacant position after stage $n$.

Example. Here again, an example will be of use. Take the diagramme $(v, t)$ with

$$
v=c_{0}^{\prime \prime} a_{0} c_{1}^{\prime} a_{1} b_{2} a_{1} c_{2}^{\prime \prime} b_{2} b_{1} \text { and } s=001021011 \text {; }
$$

then the sequence of partial trees shown in Fig. 6 is generated.

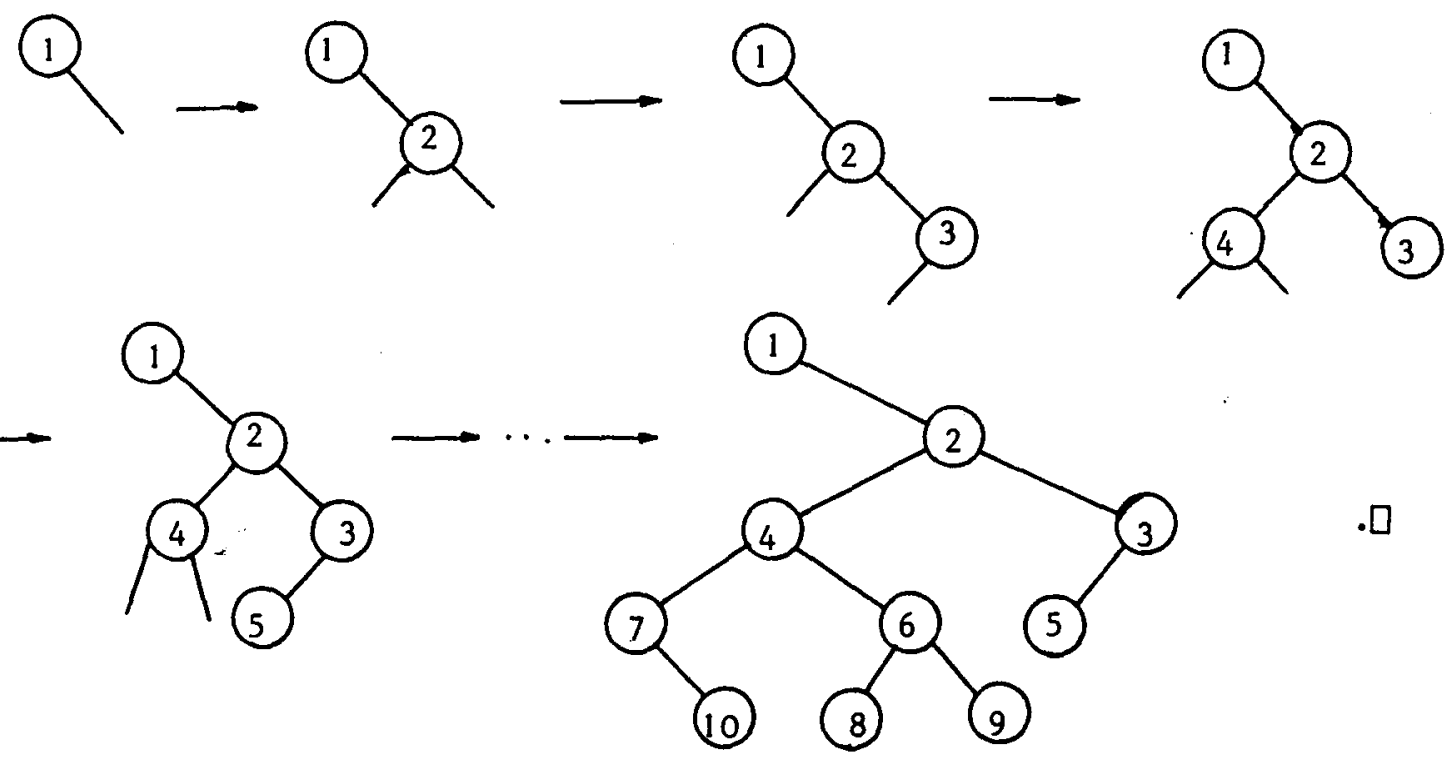

Fig. 6 
As before the correspondence is useful in connection with emuneration problems. Given a permutation $\sigma=\sigma_{1} \sigma_{2} \cdots \sigma_{n}$, element $\sigma_{j}$ said to be a maximum if $\sigma_{j-1}<\sigma_{j}>\sigma_{j+1}$; a minimum if $\sigma_{j-1}>\sigma_{j}<\sigma_{j+1}$; a double rise if $\sigma_{j-1}<\sigma_{j}<\sigma_{j+1}$; a double fall if $\sigma_{j-1}>\sigma_{j}>\sigma_{j+1}$ (conventionally $\sigma_{0}=\sigma_{n+1}=0$ ). A rise in a permutation is a value $\sigma_{j}$ such that $\sigma_{j-1}<\sigma_{j}$, i.e. a rise is either a double rise or a minimum. Obviously, the number of maxima of a permutation is equal to the number of its minima plus one.

Theorem 3A. Let $\mathscr{P}_{k, l, m}$ be the number of permutations having $k$ minima (hence $k+1$ maxima), $l$ double rises and $m$ double falls. The generating function

$$
P(u, v, w, z)=\sum P_{k, l, m} u^{k} v^{l} w^{m} z^{2 k+l+m+1}
$$

has the expression:

$$
P(u, v, w, z)=\frac{1}{1-1(v+w) z-\frac{1 \cdot 2 u z^{2}}{1-2(v+w) z-\frac{2 \cdot 3 u z^{2}}{\cdots}}} .
$$

In particular:

(i)

$$
\sum_{n \geqslant 0}(n+1) ! z^{n}=\frac{1}{1-2 z-\frac{1 \cdot 2 z^{2}}{1-4 z-\frac{2 \cdot 3 z^{2}}{\cdots}}}
$$

(ii)

$$
\sum_{n, k \geqslant 0} A_{n+1, k+1} u^{k} z^{n}=\frac{1}{1-1(1+u) z-\frac{1 \cdot 2 u z^{2}}{1-2(1+u)-\frac{2 \cdot 3 u z^{2}}{\cdots}}}
$$

(iii)

$$
\sum_{n \geqslant 0} E_{2 n+1} z^{2 n+1}=\frac{z}{1-\frac{1 \cdot 2 z^{2}}{1-\frac{2 \cdot 3 z^{2}}{1-\frac{3 \cdot 4 z^{2}}{\cdots}}}}
$$

in which $A_{n, k}$ is the Eulerian number counting the permutations of $[1 \cdots n]$ with $k$ rises and $E_{2 n+1}$ is the odd Euler number or tangent number counting the alternating permutations of $[2 n+1]$.

Proof. The proof results directly from a combination of Theorem 1 and Theorem $\mathrm{F}-\mathrm{V}:$ an $a$ in the correspondence is associated with a minimum, a $b$ with a maximum, a $c^{\prime}$ with a double fall and a $c^{\prime \prime}$ with a double rise. We thus use the morphism $\mu$

$$
\mu\left(a_{j}\right)=(j+1) u z ; \quad \mu\left(b_{k}\right)=(k+1) z ; \quad \mu\left(c_{j}^{\prime}\right)=(j+1) v z ; \quad \mu\left(c_{j}^{\prime \prime}\right)=(j+1) w z .
$$


The special cases are obtained by setting:

(i) $u=v=w=1$;

(ii) $u=w, v=1$

(iii) $v=w=1, u=1$;

and rearranging the expressions.

There is an important modification of the Françon-Viennot correspondence also considered in [13]. Let $S_{n+1}^{\prime}$ denote permutations where $(n+1)$ occurs in the last position.

$$
S_{n+1}^{\prime}=\left\{\sigma_{1} \cdots \sigma_{n+1} \mid \sigma_{n+1}=n+1\right\} .
$$

The set $S_{n+1}^{\prime}$ is obviously isomorphic to $S_{n}$. Now permutations in $S_{n+1}^{\prime}$ correspond to trees with $(n+1)$ at the bottom of the right branch. By the Françon-Viennot correspondence; they are associated to diagrammes $(u, s)$ with forbidden positions, given by

$$
\begin{array}{lll}
\text { for all } 1 \leqslant i \leqslant n, & \text { if } u_{i}=b_{k}: & s_{i} \neq k, \\
& \text { if } u_{i}=c_{j}^{\prime \prime}: & s_{i} \neq j .
\end{array}
$$

These restrictions express on the path diagramme the fact that no left branching son nor leaf different from $(n+1)$ can occur on the right branch of the tournament tree at any intermediary stage of the construction.

Theorem 3B. The following expansions hold

$$
\begin{aligned}
& \sum_{n \geqslant 0} n ! z^{n}=\frac{1}{1-z-\frac{1^{2} z^{2}}{1-3 z-\frac{2^{2} z^{2}}{\cdots}}} ; \\
& \sum_{n, k \geqslant 0} A_{n, k} u^{k} z^{n}=\frac{1}{1-u z-\frac{1^{2} u z^{2}}{1-(1+2 u) z-\frac{2^{2}}{\cdots} z^{2}}} ; \\
& \sum E_{2 n} z^{2 n}=\frac{1}{1-\frac{1^{2} z^{2}}{1-\frac{2^{2}}{z^{2}}}} ; \\
& \sum s_{n, k} u^{k} z^{n}=\frac{{\frac{3}{2} z^{2}}_{\cdots}^{\cdots}}{1-u z-\frac{u z^{2}}{1-(2+u) z-\frac{(1+u) 2 z^{2}}{\cdots}}}
\end{aligned}
$$

where the $A_{n, k}$ are the Eulerian numbers; $E_{2 n}$ is the $2 n$-th Euler number of secant number counting the alternating permutations of $[2 n] ; s_{n, k}$ is the Stirling number of the first kind counting the permutations of $[n]$ having $k$ right-to-left minima. 
Proof. From the above remarks, $S_{n}$ which is in bijection with $S_{n+1}^{\prime}$ corresponds to diagrammes relative to the possibility function:

$\operatorname{pos}\left(a_{j}\right)=j+1 ; \quad \operatorname{pos}\left(b_{k}\right)=k ; \quad \operatorname{pos}\left(c_{j}^{\prime}\right)=j ; \quad \operatorname{pos}\left(c_{j}^{\prime \prime}\right)=j+1, \quad$ for all $j \geqslant 0, k \geqslant 1$.

The result follows by choosing adequate morphisms in each case. For instance right-to-left minima correspond in a diagramme $(u, s)$ to positions $i$ such that

$$
\begin{aligned}
& u_{i}=a_{j} \quad \text { and } \quad s_{i}=j, \\
& u_{i}=c_{j}^{\prime \prime} \quad \text { and } \quad s_{i}=j \text { for some } j \geqslant 0 .
\end{aligned}
$$

The morphism which gives the Stirling numbers of the first kind is thus

$$
\mu\left(a_{j}\right)=\mu\left(c_{j}^{\prime \prime}\right)=((j-1)+u) z ; \quad \mu\left(b_{k}\right)=k z ; \quad \mu\left(c_{j}^{\prime}\right)=j z \quad \text { for } j \geqslant 0, k \geqslant 1 .
$$

Theorem 3C. Let $C_{n_{1}, n_{2}, n}$ be the number of permutations in $S_{n}$ having $n_{1}$ cycles of length 1 and $n_{2}$ cycles of length $\geqslant 2$. The generating function

$$
C\left(u_{1}, u_{2}, z\right)=\sum C_{n_{1}, n_{2}, n} u_{1}^{n_{1}} u_{2}^{n_{2}} z^{n}
$$

has the expansion

$$
C\left(u_{1}, u_{2}, z\right)=\frac{1}{1-u_{1} z-\frac{1 u_{2} z^{2}}{1-\left(2+u_{1}\right) z-\frac{2\left(1+u_{2}\right) z^{2}}{\cdots}}} .
$$

In particular for $D_{n}$ the number of permutations without fixed points:

$$
\sum D_{n} z^{n}=\frac{1}{1-\frac{1^{2} z^{2}}{1-2 z-\frac{2^{2} z^{2}}{\cdots}}} .
$$

Proof. The proof follows directly from the fundamental bijection [11, p. 13] of $S_{n}$ on itself, that exchanges smallest elements of cycles and right-to-left minima. Singleton cycles correspond to right-to-left minima that are double rises; smallest elements of non-singleton cycles correspond to right-to-left minima that are also minima.

All the quantities appearing in Theorem 3 have exponential generating functions of a simple type. We mention:

$$
\begin{aligned}
& \sum_{n, k \geq 0} A_{n, k} u^{k} \frac{z^{n}}{n !}=\frac{1-u}{1-u \mathrm{e}^{z(1-u)}} ; \\
& \sum_{n \geqslant 0} E_{2 n+1} \frac{z^{2 n+1}}{2 n+1 !}=\operatorname{tg} z ; \quad \sum_{n \geqslant 0} E_{2 n} \frac{z^{2 n}}{2 n !}=\sec z=\frac{1}{\cos z} ; \\
& \sum_{n, k \geqslant 0} s_{n, k} u^{k} \frac{z^{n}}{n !}=\exp (-u \ln (1-t))=\frac{1}{(1-t)^{u}} ;
\end{aligned}
$$




$$
\begin{aligned}
& \sum C_{n_{1}, n_{2}, n} u_{1}^{n_{1}} u_{2}^{n_{2}} \frac{z^{n}}{n !}=\frac{\exp \left(u_{1}-u_{2}\right) z}{(1-z)^{u_{2}}} \\
& \sum D_{n} \frac{z^{n}}{n !}=\frac{\mathrm{e}^{-z}}{(1-z)} .
\end{aligned}
$$

The continued fraction expansions of the two generating series of factorial numbers already known to Euler are limiting cases of Gauss' continued fraction, to which the expansion relative to the Stirling numbers also reduces. The expansions relative to the Euler and Eulerian numbers have been derived by Stieltjes by means of standard addition formulae on the corresponding exponential generating series, as an application of his basic theorem.

The construction in Theorem 3 can be further extended. Consider $r$-forests of tournament trees such that $(n+j)$ occurs at the bottom of the right branch of the $j$ th component of the forest for all $j \in[1 \cdots n]$. Such forests are bijectively associated to the class $S_{n+r}^{(r)}$ of permutations of $S_{n+r}^{\prime}$ where values $n+1, n+$ $2, \ldots, n+r$ appears as a subsequence in that order:

$$
S_{n+r}^{(1)}=\left\{\sigma_{1} \sigma_{2} \cdots \sigma_{n+2} \mid n+1=\sigma_{j_{1}} \cdots n+r=\sigma_{j_{r}} \Rightarrow j_{1}<j_{2} \cdots<j_{r}=n+r\right\} .
$$

Obviously $S_{n+1}^{(1)}$ is identical with $S_{n+1}^{\prime}$. We can modify the Françon-Viennot correspondence to see that $r$-forests of this type correspond to path diagrammes relative to the possibility function:

$$
\begin{aligned}
& \operatorname{pos}\left(a_{j}\right)=j+r ; \quad \operatorname{pos}\left(b_{k}\right)=k ; \\
& \operatorname{pos}\left(c_{j}^{\prime}\right)=j ; \quad \operatorname{pos}\left(c_{j}^{\prime \prime}\right)=j+r
\end{aligned}
$$

Notice that the cardinality of $S_{n+2}^{(2)}$ is expressed by the rising factorials:

$$
\operatorname{card}\left(S_{n+r}^{\prime(r)}\right)=(r)_{n}=r(r+1) \cdots(r+n-1) .
$$

Now parameters of tournament trees can be extended additively to forests. New parameters are thus defined on $S_{n+r}^{(r)}$. For instance starting with the double nodes and right branching simple nodes of tournament trees, the corresponding parameter on forests is the total number of double nodes and right branching simple nodes and for an $r$-forest of size $n+r$; it corresponds to the number of rises in the associated permutation of $S_{n+r}^{(r)}$ when one does not count possible rises of the type $(n+j ; n+j+1)$. Call $A_{n, k}^{(r)}$ the number of permutations of $S_{n+r}^{(r)}$ having $k$ such rises; the $A_{n, k}^{(r)}$ are the Eulerian numbers of order $r$ [21, 11]. Similarly; call $E_{2 n}^{(r)}$ the number of permutations of $S_{2 n+r}^{\prime(2)}$ such that the elements $1,2,3, \ldots, 2 n$ are either minima or maxima, i.e. no value $1,2, \ldots, 2 n$ can either be a double rise or a double fall. The $E_{2 n}^{(r)}$ are the Euler number of order $r$. We have

Proposition 9. The generating series for the rising factorials, the Eulerian and Euler 
numbers of order $r$ have the following continued fraction expansions:

$$
\begin{aligned}
& \sum_{n \geqslant 0}(r)_{n} z^{n}=\frac{1}{1-r z-\frac{1 r z^{2}}{1-(r+2) z-\frac{2(r+1) z^{2}}{\cdots}}} ; \\
& \sum_{n, k \geqslant 0} A_{n, k}^{(r)} u^{k} z^{n}=\frac{1}{1-r u z-\frac{1 r u z^{2}}{1-((r+1) u+1) z-\frac{2(r+1) u z^{2}}{\cdots}}} \\
& \sum_{n \geqslant 0} E_{2 n}^{(r)} z^{2 n}=\frac{1}{1-\frac{1 r z^{2}}{1-\frac{2(r+1) z^{2}}{\cdots}}}
\end{aligned}
$$

These enumeration results have the interest to be expressible by $r$ th powers of exponential generating functions:

$$
\begin{aligned}
& \sum_{n \geqslant 0}(r)_{n} \frac{z^{n}}{n !}=\frac{1}{(1-z)^{r}} ; \quad \sum_{n \geqslant 0} E_{2 n}^{(r)} \frac{z^{2 n}}{2 n !}=\sec ^{r} z ; \\
& \sum_{n, k \geqslant 0} A_{n, k}^{(r)} u^{k} \frac{z^{n}}{n !}=\left(\frac{1-u}{1-u \exp (z(1-u))}\right)^{r} .
\end{aligned}
$$

The $A_{n, k}^{(r)}$ are related to quantities that appear in the enumeration of permutations with restricted positions $[21,11]$. For instance they are given by

$$
A_{n, k}^{(r)}=r \cdot{ }^{r} a_{n+r, n+1-k},
$$

where the ${ }^{r} a_{n, p}$ are the numbers of [11, p. 45].

From an algebraic point of view, the continued fraction of the series of the rising factorials coincides with the expansion relative to the Stirling numbers. The continued fractions relative to the $E_{2 n}^{(r)}$ and $A_{n, k}^{(r)}$ have been computed by Stieltjes [26] and Rogers [23].

We now turn to the study of elliptic functions. The elliptic functions $c n$ and $d n$ are defined [31] by

$$
\operatorname{cn}(u, \alpha)=\cos \operatorname{am}(u, \alpha) ; \quad \operatorname{dn}(u, \alpha)=\sqrt{1-\alpha^{2} \sin ^{2} \operatorname{am}(u, \alpha)}
$$

where $\operatorname{am}(u, \alpha)$ is the inverse of an elliptic integral: by definition

$$
\operatorname{am}(u, \alpha)=\phi \quad \text { iff } \quad u=\int_{0}^{\phi} \frac{\mathrm{d} t}{\sqrt{1-\alpha^{2} \sin ^{2} t}} .
$$

The functions $\operatorname{cn}(u, \alpha)$ and $\operatorname{dn}(u, \alpha)$ have power series expansions:

$$
\begin{aligned}
& \operatorname{cn}(u, \alpha)=\sum_{n \geqslant 0}(-1)^{n-1} \frac{u^{2 n}}{2 n !} C_{n}\left(\alpha^{2}\right) ; \\
& \operatorname{dn}(u, \alpha)=\sum_{j \geqslant 0}(-1)^{n-1} \frac{u^{2 n}}{2 n !} D_{n}\left(\alpha^{2}\right),
\end{aligned}
$$


where $C_{n}$ and $D_{n}$ are polynomials of degree $n-1$ with $D_{n}$ the reciprocal polynomial of $C_{n}$. The noticeable fact about the $C_{n}$ polynomials (hence the $D_{n}$ ) is that they have positive integer coefficients. Furthermore, the coefficients of $C_{n}$ (and $D_{n}$ ) add up to the Euler number $E_{2 n}$ : this corresponds to the well known property of the elliptic functions to reduce to the hyperbolic function when the modulus $\alpha$ equals 1 . Since $E_{2 n}$ counts the number of alternating permutations over $[1 \cdots 2 n]$, the question naturally arises whether there is some natural partitioning parameter of alternating permutations enumerated by the coefficients of the $C_{n}$ 's. We prove:

Theorem 4. The coefficient $C_{n, r}$ in the expansion of the elliptic series

$$
\operatorname{cn}(u, \alpha)=\sum_{n, k \geqslant 0}(-1)^{n} C_{n, k} \alpha^{2 k} \frac{u^{2 n}}{2 n !}
$$

counts the alternating permutations over $[2 n]$ having $k$ minima of even value.

Proof. As is classically derived from the addition theorems for elliptic functions, the following expansion holds:

$$
\sum_{n, k \geqslant 0}(-1)^{n} C_{n, k} \alpha^{2 k} z^{2 n}=\frac{1}{1+\frac{1^{2} z^{2}}{1+\frac{2^{2} \alpha^{2} z^{2}}{1+\frac{3^{2} z^{2}}{1+\frac{4^{2} \alpha^{2} z^{2}}{\cdots}}}}} .
$$

Thus $C_{n, r}$ counts the number of path diagrammes of length $2 n$, relative to the possibility function:

$$
\operatorname{pos}\left(c_{j}^{\prime}\right)=\operatorname{pos}\left(c_{j}^{\prime \prime}\right)=0 ; \quad \operatorname{pos}\left(a_{j}\right)=j+1 ; \quad \operatorname{pos}\left(b_{k}\right)=k \quad \text { for all } j \geqslant 0, k \geqslant 1,
$$

whose path comprises $2 r$ letter in $\left\{a_{1}, a_{3}, a_{5}, \ldots\right\} \cup\left\{b_{2}, b_{4}, b_{6}, \ldots\right\}$.

Equivalently, $C_{n, r}$ counts diagrammes whose path has $r$ letters in $\left\{a_{1}, a_{3}, a_{5}, \ldots\right\}$, since in a path a letter $a_{2 j+1}$ is matched by a letter $b_{2 j}$. Notice also that in a path like

$$
a_{0} \underline{a}_{1} b_{2} \underline{a}_{1} a_{2} \underline{a}_{3} b_{4} s_{3} s_{2} s_{1}
$$

the $a_{1}, a_{3}, a_{5}, \ldots$ (underlined above) occur at even positions starting from the left. Using the Françon-Viennot correspondence between $S_{n+1}^{\prime}$ and diagrammes of length $n$, we see that the $a_{2 j+1}$ correspond to minima of even value.

This interpretation is distinct of the first interpretation of the coefficients of elliptic functions given by Viennot [29].

Example. If $\mathscr{C}_{n, r}$ is the set of alternating permutations of $2 n$ with $k$ even minima, 
we have:

$\mathscr{C}_{0,0}=\{0\} ; \quad \mathscr{C}_{1,0}=\{21\} ; \quad \mathscr{C}_{2,0}=\{2143\} ; \quad \mathscr{C}_{2,1}=\{4231,3142,3241,4132\}$,

which is consistent with the known values of the $C_{n, r}$.

\subsection{Cycles of binomial coefficients}

In this section, we use the results of part 1 to obtain a combinatorial proof of an expression for generating series of quantities akin to Carlitz's cycles of binomial coefficients $[3,22]$.

We consider here (planted plane) trees in the sense of Knuth [15], where each node has any number of successors. The height of a node is the distance measured in number of edges from that node to the root of the tree. A tree has specification $\left(j_{1}, j_{2}, \ldots, j_{h}\right)$ if it has size $1+j_{1}+j_{2}+\cdots+j_{h}$ and is formed with $j_{1}$ nodes of height $1, \ldots, j_{h}$ nodes of height $h$. For instance the tree in Fig. 7

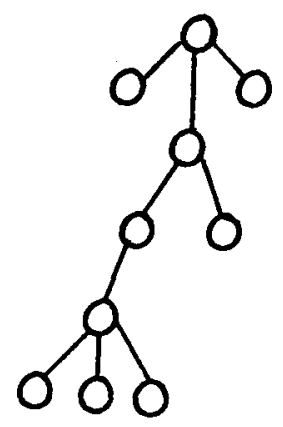

Fig. 7

has specification $(3,2,1,3)$.

Proposition $10 .^{2}$ (i) The number $\alpha\left(j_{1}, j_{2}, \ldots, j_{h}\right)$ of trees with specification $\left(j_{1}, j_{2}, \ldots, j_{h}\right)$ has generating function

$$
\sum \alpha\left(j_{1}, j_{2}, \ldots, j_{h}\right) u_{1}^{j_{1}} u_{2}^{j_{2}} \cdots u_{h}^{j_{h}}
$$

expressed by

$$
\begin{array}{r}
A^{[h]}\left(u_{1}, u_{2}, \ldots, u_{h}\right)=\frac{1}{1-\frac{u_{1}}{1-\frac{u^{2}}{\cdots}},} \\
\frac{1}{1-u_{h}}
\end{array}
$$

and its value is

$$
\alpha\left(j_{1}, j_{2}, \ldots, j_{h}\right)=\left(\begin{array}{c}
j_{1}+j_{2}-1 \\
j_{1}-1
\end{array}\right) \cdots\left(\begin{array}{c}
j_{h-1}+j_{h}-1 \\
j_{h-1}-1
\end{array}\right) .
$$

\footnotetext{
${ }^{2}$ Proposition 10(i) has been derived independently by Read [32].
} 
(ii) The number $\beta\left(i_{1}, i_{2}, \ldots, i_{h}\right)$ of trees with specification $\left(i_{1}+1, i_{2}+1, \ldots, i_{h}+\right.$ 1) having their leftmost branch of height exactly $h$ has generating function

$$
B^{[h]}\left(u_{1}, u_{2}, \ldots, u_{h}\right)=\sum \beta\left(i_{1}, i_{2}, \ldots, i_{h}\right) u_{1}^{i_{1}} u_{2}^{i_{2}} \cdots u_{h}^{i_{h}}
$$

expressed by

$$
\begin{aligned}
B^{[h]}\left(u_{1}, \ldots, u_{h}\right)=A^{[1]}\left(u_{h}\right) A^{[2]}\left(u_{h-1}, u_{h}\right) \cdots A^{[h]}\left(u_{1}, u_{2}, \ldots, u_{h}\right) \\
=\frac{1}{1-u_{h}} \cdot \frac{1}{1-\frac{u_{h-1}}{1-u_{h}}} \cdots \frac{1}{1-\frac{u_{1}}{\cdots}} \\
\frac{1}{1-u_{h}}
\end{aligned}
$$

and its value is

$$
\beta\left(i_{1}, i_{2}, \ldots, i_{h}\right)=\left(\begin{array}{c}
i_{1}+i_{2} \\
i_{1}
\end{array}\right) \cdots\left(\begin{array}{c}
i_{h-1}+i_{h} \\
i_{h-1}
\end{array}\right) .
$$

Proof. The classical correspondence between path and trees associates to a tree with specification $\left(j_{1}, j_{2}, \ldots, j_{h}\right)$ a positive path of height $h$ with $j_{k}$ occurrences of letter $a_{k-1}$ matched by $j_{k}$ occurrences of letter $b_{k}$ for all $1 \leqslant k \leqslant h$. Part (i) of the proposition follows from the expression of the Stieltjes-Rogers polynomials in Proposition 3 using the morphism $\mu\left(a_{j}\right)=u_{j+1} ; \mu\left(s_{k}\right)=1$.

The same correspondence associates to a tree with specification $\left(i_{1}+1 ; i_{2}+\right.$ $\left.1 ; \ldots ; i_{h}+1\right)$ and with leftmost branch of length $h$, a path from $h$ to 0 having height $h$. In such a path we can single out the rightmost occurrences of letter $s_{k}$ for all $k: 1 \leqslant k \leqslant h$, which yields the factorization

$$
w=s_{h} v_{h-1} s_{h-1} v_{h-2} s_{h-2} \cdots v_{1} s_{1} v_{0} .
$$

Thus the series of the $u$ 's factorizes into a product. Writing $W$ for the characteristic series of the $w$ corresponding to our description, we have

$$
W=s_{h} V_{h-1} s_{h-1} \cdots V_{1} s_{1} V_{0}
$$

where $V_{j}$ is the characteristic series of paths from $j$ to $j$ with height $\leqslant h$ and such that all their points have height $\geqslant j$. Thus

$$
\begin{array}{r}
\mu\left(V_{j}\right)=\frac{1}{1-\frac{u_{j+1}}{1-\frac{u_{j+2}}{\cdots}}} \\
\frac{1}{1-u_{h}}
\end{array}
$$

from which the product expression of $B^{[h]}$ is derived.

The closed form expression for $\beta\left(i_{1}, i_{2}, \ldots, i_{n}\right)$ follows from a straight forward modification of the counting argument of Proposition 3. 
Anticipating on some of the developments of Section 3, we can see that the successive numerators and denominators in the product giving $B^{[h]}$ simplify, leaving only the last denominator standing in the expression, so that

$$
B^{[h]}\left(u_{1}, u_{2}, \ldots, u_{h}\right)=\frac{1}{\left[-u_{1},-u_{2}, \ldots,-u_{h}\right]}
$$

where the cumulants $\left[x_{1}, x_{2}, \ldots, x_{n}\right]$ are defined recurrently by

$$
\begin{aligned}
& {[]=1 ; \quad\left[x_{1}\right]=1+x_{1} ;} \\
& {\left[x_{1}, x_{2}, \ldots, x_{n}\right]=\left[x_{1}, x_{2}, \ldots, x_{n-1}\right]+\left[x_{1}, x_{2}, \ldots, x_{n-2}, x_{n}\right] .}
\end{aligned}
$$

From this follows the equality:

where

$$
B^{[h]}\left(u_{1}, u_{2}, \ldots, u_{h}\right)=\frac{1}{a u_{1} u_{n}+b u_{1}+c u_{n}+d},
$$

$$
\begin{aligned}
& a=\left[-u_{3}, \ldots,-u_{n-2}\right] ; \quad b=-\left[-u_{3}, \ldots,-u_{n-1}\right] ; \\
& c=-\left[-u_{2}, \ldots,-u_{n-2}\right] ; \quad d=\left[-u_{2}, \ldots,-u_{n-1}\right] .
\end{aligned}
$$

This expression is essentially Carlitz's result [2].

\section{Enumerative properties of convergents}

\subsection{Convergents}

Starting with a $J$-fraction

$$
J(z)=\frac{1}{1-c_{0} z-\frac{a_{0} b_{1} z^{2}}{1-c_{1} z-\frac{a_{1} b_{2} z^{2}}{\cdots}}}
$$

we define the $h$ th convergent as the (finite) fraction

$$
J^{[h]}(z)=\frac{1}{1-c_{0} z-\frac{a_{0} b_{1} z^{2}}{\frac{\cdots}{1-c_{h} z}} .}
$$

The $h$ th convergent corresponds to paths with height $\leqslant h$ (see Section 1 ) and allowing commutativity of indeterminates, one has classically

$$
J^{[h]}(z)=P_{h}(z) / Q_{h}(z)
$$

where $P_{h}$ and $Q_{h}$ are polynomials which satisfy a linear recurrence

$$
\begin{aligned}
& P_{-1}(z)=0 ; \quad P_{0}(z)=1 ; \\
& P_{h}(z)=\left(1-c_{h} z\right) P_{h-1}(z)-a_{h-1} b_{h} z^{2} P_{h-2}(z) ; \\
& Q_{-1}(z)=1 ; \quad Q_{0}(z)=1-c_{0} z ; \\
& Q_{h}(z)=\left(1-c_{h} z\right) Q_{h-1}(z)-a_{h-1} b_{h} z^{2} Q_{h-2}(z) .
\end{aligned}
$$

Here we have set conventionally $J^{[-1]}=0=0 / 1$. 
The polynomials $P$ and $Q$ appear in a number of enumeration results relative to paths.

First consider the paths of height exactly equal to $h$; these have generating function

$$
J^{[h]}=J^{[h-1]}=\frac{P_{h}}{Q_{h}}-\frac{P_{h-1}}{Q_{h-1}}=\frac{P_{h} Q_{h-1}-P_{h-1} Q_{h}}{Q_{h} Q_{h-1}} .
$$

Using the classical determinant identity, we have

$$
P_{h} Q_{h-1}-P_{h-1} Q_{h}=a_{0} a_{1} \cdots a_{h-1} b_{1} b_{2} \cdots b_{h} z^{2 h},
$$

hence

$$
J^{[h]}-J^{[h-1]}=\frac{\lambda_{h} z^{2 h}}{Q_{h} Q_{h-1}}, \text { with } \lambda_{h}=\left(a_{0} b_{1}\right) \cdots\left(a_{h-1} b_{h}\right) .
$$

This result is consistent with the fact that $J^{[h]}-J^{[h-1]}$ counts paths of height exactly $h$, which have necessarily length $\geqslant 2 h$.

Consider now paths from height $h$ to height $h$ whose elements are all at height $\geqslant h$. Application of Theorem 1 shows that the corresponding generating function denoted $J^{/ h /}$ has the expansion

$$
J^{\prime h /}=\frac{1}{1-c_{h} z-\frac{a_{h} b_{h+1} z^{2}}{1-c_{h+1}-\frac{a_{h+1} b_{h+2} z^{2}}{\cdots}} ;}
$$

we shall call it the $h$ th truncation of the continued fraction $J$. The truncation $J^{/ h /}$ is expressible by

$$
J^{\prime 0 /}(z)=J(z) ; \quad J^{\prime h /}(z)=\frac{1}{a_{h-1} b_{h} z^{2}} \frac{Q_{h-1}(z) J(z)-P_{h-1}(z)}{Q_{h-2}(z) J(z)-P_{h-2}(z)} .
$$

Finally consider the generating series $K_{h}(z)$ of the paths from height 0 to height $h$; obviously

$$
K_{h}(z)=J^{\prime 0 /}(z) \cdot a_{0} z J^{\prime 1 /}(z) \cdot a_{1} z \cdots J^{/ h /}(z),
$$

so that

$$
K_{h}(z)=\frac{1}{b_{1} b_{2} \cdots b_{h} z^{h}}\left(Q_{h-1}(z) J(z)-P_{h-1}(z)\right) .
$$

In particular, paths from 0 to $h$ with height $h$ have a generating function denoted $K_{h}^{[h]}$, whose expression is obtained by replacing $J(z)$ by $P_{h} / Q_{h}$ in the expression above:

$$
K_{h}^{[h]}(z)=\frac{1}{b_{1} b_{2} \cdots b_{h} z^{h}}\left(Q_{h-1}(z) \frac{P_{h}(z)}{Q_{h}(z)}-P_{h-1}(z)\right) ;
$$

hence using again the determinant identity:

$$
K_{h}^{[h]}(z)=\frac{a_{0} a_{1} \cdots a_{h-1} z^{h}}{Q_{h}(z)} .
$$


We thus see that the numerator polynomials $P_{h}$, and the denominator polynomials $Q_{h}$ appear in several enumeration formulae relative to generalized paths. The denominator polynomials usually have simpler expressions. The numerator polynomials $P_{h}$ can be expressed by convolution of $J(z)$ with $Q_{h}(z)$ as follows: for each $m \geqslant 0$, define the erasing operator $E_{m}$ as a linear operator from $C[[z]]$ into $C[z]$ satisfying:

$$
E_{m}\left(z^{n}\right)=\left\{\begin{array}{cc}
0 & \text { if } n>m \\
z^{n} & \text { if } n \leqslant m .
\end{array}\right.
$$

As is readily checked $\operatorname{val}\left(K_{h}(z)\right)=h$, and thus $\operatorname{val}\left(Q_{h-1}(z) J(z)-P_{h-1}(z)\right)=2 h$; the polynomial $P_{h-1}$ of degree $h-2$ thus coincides with the first $h-1$ terms in the product $Q_{h-1}(z) J(z)$; hence, shifting indices, we get

$$
P_{h}(z)=E_{h-1}\left(Q_{h}(z) J(z)\right) .
$$

\subsection{Inversion relations}

We have seen the identity:

$$
K_{h}(z)=\frac{1}{b_{1} b_{2} \cdots b_{h} z^{h}}\left(Q_{h-1}(z) J(z)-P_{h-1}(z)\right),
$$

where val $K_{h}(z)=h$. Thus,

$$
\operatorname{val}\left(Q_{h-1}(z) J(z)-P_{h-1}(z)\right)=2 h .
$$

This property can be rephrased as an orthogonality relation (see e.g. [30, p. 193]): Let $J(z)=\sum_{n \geqslant 0} R_{n} z^{n}$ be the power series expansion of $J$; a linear form over $C[z]$ is defined by

$$
\left\langle x^{n}\right\rangle=R_{n}
$$

it can be extended to a bilinear form over $C[z]$ by setting

$$
\left\langle x^{m} \mid x^{n}\right\rangle=\left\langle x^{m+n}\right\rangle=R_{m+n} .
$$

Now let

$$
Q_{h-1}(z)=\sum_{0 \leqslant r \leqslant h} Q_{h-1, r} z^{r}
$$

and define the reciprocal polynomials:

$$
\bar{Q}_{h-1}(z)=z^{h} Q_{h-1}\left(\frac{1}{z}\right)=\sum Q_{h-1, h-r} z^{r}
$$

The $\bar{Q}_{h-1}$ are normalized (the coefficient of $z^{h}$ is 1 ) and we have

$$
\begin{aligned}
\left\langle z^{n} \mid \bar{Q}_{h-1}(z)\right\rangle & =\left\langle z^{n} \bar{Q}_{h-1}(z)\right\rangle=\left\langle\sum Q_{h-1, h-r} z^{n+r}\right\rangle \\
& =\sum_{0 \leqslant r \leqslant h} R_{n+r} Q_{h-1, h-r} \\
& =\operatorname{coeff}\left(J(z) Q_{h-1}(z) ; z^{h+n}\right) .
\end{aligned}
$$

From the preceding remarks, we see that

$$
\left\langle z^{n} \mid \bar{Q}_{h-1}(z)\right\rangle=0 \quad \text { if } 0 \leqslant n \leqslant h=\operatorname{deg}\left(\bar{Q}_{h-1}\right) .
$$


Notice also for $n \geqslant h$ the relations for $K_{h}(z)=\sum_{n \geqslant 0} k_{h, n} z^{n}$ :

$$
k_{h, n}=\frac{1}{b_{1} b_{2} \cdots b_{h}}\left\langle z^{n} \mid \bar{Q}_{h-1}(z)\right\rangle
$$

and in particular

$$
\left\langle z^{h} \mid z^{h}\right\rangle=\left\langle z^{h} \mid \bar{Q}_{h-1}(z)\right\rangle=b_{1} b_{2} \cdots b_{h} k_{h h}=\left(a_{0} b_{1}\right) \cdots\left(a_{h-1} b_{h}\right) .
$$

Thus the polynomials $\left\{\bar{Q}_{h-1}\right\}_{h \geqslant 0}$ form an orthogonal family of polynomials with respect to the bilinear form associated to $J$. The change of basis from the $\left\{z^{n}\right\}_{n \geqslant 0}$ to the $\left\{\bar{Q}_{h-1}\right\}_{h \geqslant 0}$ is thus described by

$$
\left\{\begin{aligned}
\bar{Q}_{h-1}(z)= & \sum_{0 \leqslant r \leqslant h} Q_{h-1, h-r} z^{r}, \\
z^{n}= & \sum_{0 \leqslant h \leqslant n} \frac{\left\langle z^{n} \mid Q_{h-1}(z)\right\rangle}{\left\langle\bar{Q}_{h-1}(z) \mid \bar{Q}_{h-1}(z)\right\rangle} Q_{h-1}(z)=\sum \frac{k_{h, n}}{a_{0} a_{1} \cdots a_{h-1}} \bar{Q}_{h-1} .
\end{aligned}\right.
$$

We thus obtain:

Proposition 11. The Stieltjes matrix of extended paths normalized by columns:

$$
\bar{S}=\left(\bar{s}_{h, n}\right) \quad \text { with } \bar{s}_{h, n}=\frac{k_{h, n}}{a_{0} a_{1} \cdots a_{h-1}},
$$

and the matrix of the coefficients of the denominator polynomials:

$$
\bar{Q}=\left(\bar{q}_{h, r}\right) \quad \text { with } \bar{q}_{h, r}=Q_{h-1, h-r},
$$

are inverse of each other.

Example. The first elements of the $\bar{Q}$ and $\bar{S}$ matrices are given below when $c_{j}=0$ for all $j$.

$$
\begin{gathered}
\bar{S}_{5 \times 5}=\left(\begin{array}{ccccc}
1 & & & & \\
0 & 1 & & & \\
\lambda_{0} & 0 & 1 & \\
0 & \lambda_{0}+\lambda_{1} & 0 & 1 & \\
\lambda_{0}^{2}+\lambda_{0} \lambda_{1} & 0 & \lambda_{0}+\lambda_{1}+\lambda_{2} & 1
\end{array}\right) \\
\bar{Q}_{5 \times 5}=\left(\begin{array}{ccccc}
1 & 1 & & & \\
0 & 0 & 1 & & \\
-\lambda_{0} & 0 & 0 & 1 & \\
0 & -\lambda_{0}-\lambda_{1} & 0 & & \\
\lambda_{0} \lambda_{1} & 0 & -\lambda_{0}-\lambda_{1}-\lambda_{2} & 0 & 1
\end{array}\right) .
\end{gathered}
$$

Here we have set $\lambda_{j}=a_{j} b_{j+1}$. 
The elements of the $\vec{S}$ matrix can still be interpreted as extended paths whose falls only are marked with the $\lambda_{j}$ 's. The elements of the $\bar{Q}$ matrix also represent certain types of paths in a lattice, as can be seen from the recurrence:

$$
Q_{h, r}=Q_{h-1, r}-c_{h} Q_{h-1, r-1}-\lambda_{h-1} Q_{h-2, r-2},
$$

or equivalently:

$$
\bar{q}_{h, r}=\bar{q}_{h-1, r-1}-c_{h} \bar{q}_{h-1, r}-\lambda_{h-1} \bar{q}_{h-2, r}
$$

In particular when $c_{j}=0$ for all $j$ apart from the sign, the coefficient $\bar{q}_{h, r}$ corresponds to paths in the $x-y$ plane with steps given by $\left.\alpha\right|_{1} ^{1}$ and $\left.\beta\right|_{2} ^{0}$ starting from the line $y=x$. This observation is exactly the Euler-Mindig interpretation of the convergents. Recently G. Viennot (private communication to the author) has given a combinatorial proof of these inversion relations based on direct path manipulations.

\subsection{Specific cases}

Each system of path diagrammes has an associated family of polynomials. Combining results from the last section with geometrical interpretations in Section 2 , we obtain a few combinatorial interpretations for inverses and quotients of classical polynomials.

Proposition 12. Let $M_{n}^{[h]}$ be the number of paths of height $h$ and length $n$, and let $C_{n}^{[h]}$ be the number of paths of height $h$ without level steps, we have

$$
\sum_{n \geqslant 0} M_{n}^{[h]} z^{n}=\frac{Q_{h-1}(z)}{Q_{h}(z)} \text { and } \sum_{n \geqslant 0} C_{n}^{[h]} z^{n}=\frac{Q_{h-1}^{\prime}(z)}{Q_{h}^{\prime}(z)},
$$

where

(i) $Q_{h}(z)=\left(\rho^{h+3}-\bar{\rho}^{h+3} /(\rho-\bar{\rho})\right.$ with $\rho$, $\bar{\rho}$ the roots of the equation $y^{2}-(1-z) y+$ $z^{2}=0$;

(ii) $Q_{h}^{\prime}(z)=\left(\rho^{\prime h+3}-\bar{\rho}^{\prime h+3}\right) /(\rho-\bar{\rho})$ with $\rho^{\prime}, \bar{\rho}^{\prime}$ the roots of the equation $y^{2}-y+$ $z^{2}=0$.

The obvious proof is omitted. The polynomials $Q_{h}^{\prime}$ are elementary variants of the Tchebycheff polynomials. These classical results have been first established by Kreweras $[16,17]$ who also found the expression of $K_{h}^{[h]}$ in this case. The result appears in [1] under a different form.

We now come to partitions. Given a partition $\pi$ of [n], define the width of $\pi$ at $x$ denoted $\omega(\pi, \chi)$ as the number of classes overlapping $x$, that is having (at least) one element $\leqslant x$ and (at least) one element $>x$; define the width of $\pi$ denoted by $\omega(\pi)$ by:

$$
\omega(\pi)=\max _{x \in[n]}\{\omega(\pi, x)\}
$$


For instance with $\pi=\{1,7,11\}\{2,4,6,9\}\{3\}\{5,10\}\{8\}\{12,13\}$, the width of $\pi$ at 6 is equal to $3 \omega(\pi, 6)=3$ since there are exactly 3 classes overlapping 6 : $\{1,7,11\},\{2,4,6,9\}$ and $\{5,10\}$; similarly $\omega(\pi, 3)=2$ and $\omega(\pi)=3$.

Theorem 5A. Let $B_{n}^{[h]}$ be the number of partitions of $[n]$ of width $\leqslant h ;$ let $\hat{B}_{n+h}^{[h]}$ denote the number of partitions of $[n+h]$ of width $h$ such that $1,2, \ldots, h$ belong to different non-singleton classes, then

$$
\sum_{n \geqslant 0} B_{n}^{[h]} z^{n}=\frac{P_{h-1}(z)}{Q_{h-1}(z)} \text { and } \sum_{m \geqslant 0} \hat{B}_{m}^{[h]} z^{m}=\frac{h ! z^{h}}{Q_{h-1}(z)},
$$

where $Q_{h-1}(z)$ is the $h$ th reciprocal Charlier polynomial, and $P_{h-1}(z)$ is determined by the rules of Section 3.1.

Proof. The $h$ th Charlier polynomial is defined by

$$
C_{h}(z)=\sum_{0 \leqslant k \leqslant n}(-1)^{n-k}\left(\begin{array}{l}
n \\
k
\end{array}\right) x(x-1) \cdots(x-k+1),
$$

and has exponential generating function

$$
\sum_{h \geqslant 0} C_{h}(z) \frac{u^{h}}{h !}=\mathrm{e}^{-u}(1+u)^{x} .
$$

The corresponding three-term recurrence is

$$
C_{h}(z)=(z-h) C_{h-1}(z)-(h-1) C_{h-2}(z), \quad h \geqslant 2,
$$

with $C_{0}=1 ; c_{1}=x-1$. From this immediately follows that $Q_{h-1}(z)=z^{h} C_{h}(1 / z)$ for $h \geqslant 0$. Thus the polynomials $Q_{h}$ are denominator polynomials associated to the continued fraction of Theorem 2 . We complete the proof by checking that the geometrical correspondence between path diagrammes and set partitions transforms the height of the diagramme into the width of the partition.

Involutions being special cases of partitions, the notion of width applies equally well to them. The width of an involution is thus a measure of the overlap of its cycles.

Theorem 5B. Let $I_{n}^{[h]}$ be the number of involutions of $[n]$ having width $\leqslant h$; let $I_{n+h}^{[h]}$ denote the number of involutions of $[n+h]$ having width $h$ and such that $1,2, \ldots, h$ belong to different cycles of length 2 . Let $I_{n}^{,[h]}$ and $\hat{I}_{n}^{[h]}$ be the corresponding quantities relative to involutions without fixed points. Then

$$
\begin{aligned}
& \sum_{n \geqslant 0} \tilde{I}_{n}^{[h]} z^{n}=\frac{P_{h-1}(z)}{Q_{h-1}(z)} \quad \text { and } \quad \sum_{m \geqslant 0} \hat{I}_{m}^{[h]} z^{m}=\frac{h ! z^{h}}{Q_{h-1}(z)}, \\
& \sum_{n \geqslant 0} I_{n}^{\prime[h]} z^{n}=\frac{P_{h-1}^{\prime}(z)}{Q_{h-1}^{\prime}(z)} \quad \text { and } \quad \sum_{m \geqslant 0} \hat{I}_{m}^{[h] z_{z} m}=\frac{h ! z^{h}}{Q_{h-1}^{\prime}(z)},
\end{aligned}
$$


where $Q_{h-1}^{\prime}(z)=z^{h} H_{h}(1 / z)$ is the $h$ th reciprocal Hermite polynomial and $Q_{h+1}(z)=z^{h} H_{h}(1 / z-1)$. The numerator polynomials $P_{h-1}$ and $P_{h-1}^{\prime}$ are given by the rules of Section 3.1.

Proof. We only need to identify the denominator polynomials. The $m$ th Hermite polynomial is defined by

$$
H_{m}(z)=\sum_{0 \leq k \leq m / 2} \frac{m !}{k !(m-2 k) !}(-1)^{k} \frac{z^{m-2 k}}{2^{k}},
$$

and has the exponential generating function

$$
\sum H_{m}(z) \frac{u^{m}}{m !}=\mathrm{e}^{t u-u^{2} / 2}
$$

The corresponding three term recurrence relation is

$$
H_{h}(z)=z H_{h-1}(z)-(h-1) H_{h-2}(z), \quad h \geqslant 2,
$$

with $H_{0}(z)=1 ; H_{1}(z)=z$, which shows $Q_{h-1}^{\prime}$ to be identical to the reciprocal polynomial of $H_{h}(z)$.

The convergents of the continued fraction relative to the series of the factorial and secant number can also receive combinatorial interpretations.

Given a permutation $\sigma \in \mathscr{S}_{n}$, and a value $x \in[0 \cdots n]$ we consider the word $w(x) \in\{+,-\}^{n}$ called the signature of $x$ in $\sigma$ defined by

$$
(w(x))_{i}= \begin{cases}+ & \text { if } \sigma_{i}>x, \\ - & \text { if } \sigma_{i} \leqslant x .\end{cases}
$$

A cluster in a word $w \in\{+,-\}$ is a maximal factor of $w$, formed with + symbols only. We can then define the clustering of $\sigma$ at $x$ denoted by $\operatorname{cl}(\sigma, x)$ as the number of clusters in $w(x)$.

Thus with $\sigma=649315827$, the signatures $w(0), w(1), \ldots$ are

$$
\begin{aligned}
& w(0)=+++++++++, \\
& w(1)=++++-++++, \\
& w(2)=++++-++-+, \\
& w(3)=+++--++-+, \\
& w(4)=+-+--++-+, \\
& w(5)=+-+--+-+, \\
& w(6)=--+--+-+, \\
& w(7)=--+---+--, \\
& w(8)=--+-----, \\
& w(9)=-------.
\end{aligned}
$$


The clustering of $\sigma$ is defined as

$$
\operatorname{cl}(\sigma)=\max _{x} \operatorname{cl}(\sigma, x) .
$$

In the last example, the clustering of the permutation is equal to 4 .

The clustering of a permutation measures the amount of scattering of consecutive elements in the permutation. We have

Theorem 6. Let $F_{n}^{[h]}$ be the number of permutations in $S_{n}$ having clustering $<h$; let $E_{2 n}^{[h]}$ be the number of alternating permutations in $S_{2 n}$ having clustering $<h$; then

$$
\sum_{n \geqslant 0} F_{n+1}^{[h]} z^{n}=\frac{P_{h}(z)}{Q_{h}(z)} \quad \text { and } \quad \sum_{n \geqslant 0} E_{2 n}^{[h]} z^{2 n}=\frac{K_{h}^{\prime}(z)}{N_{h}(z)},
$$

where $Q_{h}(z)$ is the $h$ th reciprocal Laguerre polynomial of order 1 , and $N_{h}(z)$ is the $h$ th reciprocal Meixner polynomial; $P_{h}$ and $K_{h}$ are determined from $Q_{h}$ and $N_{h}$ by the rules of Section 3.1.

Proof. We define the $m$ th Laguerre polynomial of order 1 by

$$
L_{m}^{(1)}(z)=\sum_{0 \leqslant k \leqslant n}\left(\begin{array}{l}
n+1 \\
k+1
\end{array}\right) \frac{n !}{k !}(-1)^{n-k} z^{k}
$$

or equivalently by means of the generating series:

$$
\sum_{m \geqslant 0} L_{m}^{(1)}(z) \frac{u^{m}}{m !}=\frac{1}{(1+u)^{2}} \exp \frac{z u}{(1+u)} .
$$

It satisfies the three term recurrence relation:

$$
L_{m}^{(1)}(z)=(z-2 m) L_{m-1}^{(1)}(z)-m(m-1) L_{m-2}(z), \quad \text { with } L_{0}=1 ; L_{1}=z-2 .
$$

The Meixner polynomial $M_{h}$ have generating function $[18,4]$ :

$$
\sum_{h \geqslant 0} M_{h} \frac{z^{h}}{h !}=\left(1+t^{2}\right)^{-1 / 2} \exp (x \operatorname{arctg} t),
$$

and can also be expressed as terminating hypergeometric functions. They satisfy the three term recurrence relation:

$$
M_{h+1}(z)=z M_{h}(z)-h^{2} M_{h-1}(z), \quad \text { with } M_{0}=1 ; M_{1}=z .
$$

It is readily checked that in the Françon-Viennot correspondence, the clustering of a permutation differs by 2 from the height of the associated path diagramme. We thus obtain the generating function of the $\left\{E_{n}^{[h]}\right\}_{n \geqslant 0}$ and $\left\{F_{n}^{[h]}\right\}_{n \geq 0}$ by taking the convergents of the continued fractions of path of unbounded height. Comparison of the three term recurrence relations with those given above completes the proof of the theorem. 
In [10], we make explicit the inversion relations corresponding to these four classes of polynomials and use them to compute in a simple way the double generating functions of the elements of the Stieltjes matrices. These relations have close relationships to the Meixner classification of orthogonal polynomials [18].

\section{Conclusion}

A natural way of continuing this work is to look for other classes of combinatorial objects in correspondence with systems of path diagrammes. Notice for instance that almost all the continued fraction expansions considered here have coefficients at level $k$ that are linear in $k$. Natural candidates for such an extension are integer partitions and sequences. As shown elsewhere [33], continued fractions are also a natural way of introducing various classes of $q$-generalisations whose study should hopefully prove of interest.

Also a deeper investigation of the combinatorics of the addition formulae à la Rogers might be of interest. Our combinatorial interpretation of the coefficients of the elliptic functions $c n, d n$ ultimately relies on the addition theorems for these functions.

Finally the Stieltjes matrix and the matrix formed with the coefficients of the denominator polynomials should be made explicit in each case. In [10], we use the inversion formulae relative to the Meixner class of polynomials to derive an integral expression for a linear transform over $C[[x]]$ associated to the Stieltjes matrix of some of the continued fractions introduced in Section 2. The result is related to the analysis of the behaviour of dynamic data structures in Computer Science [10].

\section{Acknowledgements}

This work has benefited from discussions, encouragements and suggestions by $\mathrm{J}$. Françon, J. Giraud, J-M. Steyaert, G. Viennot and J. Vuillemin.

\section{References}

[1] N. de Bruijn, D. Knuth and S.O. Rice, The average height of planted plane trees, in: R.C. Read, ed., Graph Theory and Computing (Academic Press, New-York, 1972) 15-22.

[2] L. Carlitz, A binomial identity arising from a sorting problem, SIAM Rev. 6 (1964) 20-30.

[3] L. Carlitz, $q$-analog of the Lagrange expansion, in: Eulerian Series and Applications (Pennsylvania State University, 1974).

[4]. T.S. Chihara, An Introduction to Orthogonal Polynomials (Gordon and Breach, New-York, 1978).

[5] L. Comtet, Analyse Combinatoire, Vol. 2, (P.U.F., Paris, 1970).

[6] R. Cori, Un code pour les graphes planaires et ses applications, Astérisque 27 (1975). 
[7] D. Dumont, Une nouvelle interprétation combinatoire des nombres tangents, 5-th Hungarian Conference on Combinatoircs, Keszthely (1976).

[8] S. Eilenberg, Automata, Languages and Machines, Vol. A (Academic Press, New-York, 1974).

[9] P. Flajolet, Analyse d'algorithmes de manipulation de fichiers, Rapport IRIA, Rocquencourt (1978).

[10] P. Flajolet, J. Françon and J. Vuillemin, Analysis of data structures under sequences of operations (in preparation).

[11] D. Foata and M.P. Schützenberger, Théorie Géométrique des polynômes Euleriens, Lecture Notes in Math., Vol. 138 (Springer Verlag, Berlin, 1970).

[12] J. Françon, Histoires de Fichiers, RAIRO Inf. Th. 12 (1978) 49-62.

[13] J. Françon and G. Viennot, Permutations selon les pics, creux, doubles montées, doubles descentes, nombres d'Euler et nombres de Genocchi; Discrete Math. 28 (1979) 21-35.

[14] D.M. Jackson, Some results on "Product-weighted lead codes", J. Combinatorial Theory (Ser. A) 25 (1978) 181-187.

[15] D. Knuth, The Art of Computer Programming: Fundamental Algorithm (Vol. 1) (Addison Wesley, Reading, MA, 1968).

[16] G. Kreweras, Sur une classe de problèmes de dénombrement liés au treillis de partition des entiers, Cahiers du B.U.R.O. 6 (Paris, 1965).

[17] G. Kreweras, Sur les éventails de segments, Cahiers du B.U.R.O. 15 (Paris, 1970) 1-41.

[18] J. Meixner, Orthogonale Polynomsysteme mit einem besonderen Gestalt der erzeugenden Funktion, J. Lond. Math. Soc. 9 (1934) 6-13.

[19] O. Perron, Die Lehre von den Kettenbrüchen, Vol. 2 (Teubner, Stuttgart, 1954).

[20] G. Raney, Functional composition patterns and power series reversion, Trans. Am. Math. Soc. 94 (1960) 441-451.

[21] J. Riordan, An Introduction to Combinatorial Analysis (Wiley, New-York, 1958).

[22] J. Riordan, Combinatorial Identities (Wiley, New-York, 1968).

[23] L.J. Rogers, On the representation of certain asymptotic series as continued fractions, Proc. Lond. Math. Soc. (2) 4 (1907) 72-89.

[24] J. Rosen, The number of product-weighted lead codes for ballots and its relation to the Ursell function of the linear Ising model, J. Combinatorial Theory (Ser. A) 20 (1976) 377-384.

[25] M.P. Schützenberger, On context-free languages and pushdown automata, Inf. and Control 6 (1963) 246-264.

[26] T.J. Stieltjes, Sur la réduction en fraction continue d'une série procédant suivant les puissances descendantes d'une variable, Ann. Fac. Sc. Toulouse 3 (1889) 1-17.

[27] V. Strehl, Enumeration of alternating permutations according to peak sets, J. Combinatorial Theory (Ser. A) 24 (1978) 238-240.

[28] J. Touchard, Sur un problème de configurations et sur les fractions continues, Canad. J. Math. 4 (1952) 2-25.

[29] G. Viennot, Une interprétation combinatoire des dévelopements en série entière des fonctions elliptiques de Jacobi (to appear).

[30] H.S. Wall, Analytic Theory of Continued Fractions (Chelsea, New-York, 1967).

[31] E.T. Whittaker and G.N. Watson, A Course of Modern Analysis (Cambridge Univ. Press, Cambridge, 4th ed., 1927).

[32] R.C. Read, The chord intersection problem, Annals of New-York Ac. of Sc. 319 (1979) 444-454.

[33] P. Flajolet, Analyse d'algorithmes de manipulation d'arbres et de fichiers, Thèse, Fac. Sc. Orsay (1979). 